\title{
Polarized deep-inelastic lepton scattering from the polarized two- and three-nucleon bound states
}

\author{
R.-W. Schulze ${ }^{*}$ and P. U. Sauer \\ Institute for Theoretical Physics, University Hannover, D-30167 Hannover, Germany
}

(Received 15 August 1996)

\begin{abstract}
A relativistic description of polarized deep-inelastic lepton scattering from polarized nuclei is presented. It is based on front form dynamics. Convolution formulas for the nuclear spin structure functions $g_{1}^{A}$ and $g_{2}^{A}$ are derived. They require the front form spin distributions of nuclei and the nucleonic spin structure functions. The description is applied to the deuteron and the trinucleon bound states. The numerical calculations show that relativistic effects arising from the consistent quantum mechanical treatment of spin are small. Simple approximations to the convolution formulas for $g_{1}^{A}$ and $g_{2}^{A}$ are justified. The approximative convolution formulas allow the subtraction of nuclear effects from measured spin structure functions and therefore the experimental determination of the neutron spin structure functions. Polarized ${ }^{3} \mathrm{He}$ turns out to be a rather reliable effective neutron spin target for deep-inelastic scattering of polarized leptons. The differences between descriptions based on front form and on instant form dynamics are also discussed. [S0556-2813(97)03908-3]
\end{abstract}

PACS number(s): 25.30.-c, 24.70.+s, 25.10.+s, 29.25.Pj

\section{INTRODUCTION}

When ${ }^{3} \mathrm{He}$ is described as a system of three nucleons interacting through instantaneous potentials, the total angular momentum of ${ }^{3} \mathrm{He}$ is carried to a large extent by the neutron. Employing realistic two-nucleon potentials the calculated neutron contribution to the nuclear spin ranges [1] from 0.85 to 0.90 . This theoretical observation motivated experimentalists [2] to use polarized ${ }^{3} \mathrm{He}$ targets as substitutes for unavailable neutron spin targets. The tacid assumption is that the impurities present in the substitute neutron spin targets, i.e., the unwanted contributions to the ${ }^{3} \mathrm{He}$ total angular momentum arising from the proton spins and from the nucleonic orbital motion will not blur the neutron properties one would like to see. Reference [3] discusses an unfavorable case: In inclusive quasielastic scattering of polarized electrons from polarized ${ }^{3} \mathrm{He}$ [4-9], the spin-dependent response $R_{T L^{\prime}}$ carries some information on the poorly known neutron charge form factor. However, the neutron signal in $R_{T L^{\prime}}$ remains weak and gets overwhelmed by the proton one at small momentum transfers. The neutron charge form factor is small compared to the proton one and therefore contributes little, despite the fact that the neutron makes up most of the ${ }^{3} \mathrm{He}$ total angular momentum.

This paper discusses deep-inelastic scattering of polarized leptons from polarized ${ }^{3} \mathrm{He}$. Experiments measure the beam asymmetry, in particular $[10,11]$ the ${ }^{3} \mathrm{He}$ spin structure function $g_{1}^{A}$; from $g_{1}^{A}$ the neutron contribution should get unfolded and the neutron spin structure function $g_{1}^{n}$ should get extracted. The conclusion of the paper will be that indeed the neutron spin structure function $g_{1}^{n}$ can successfully be obtained in these experiments. In deep-inelastic lepton scattering polarized ${ }^{3} \mathrm{He}$ is a reliable neutron spin target in contrast to the experience of Ref. [3]. However, the theoretical discussion of the paper will often be kept general in order to

*Present address: Scandpower GmbH, Flotowstraße 41-43, D22083 Hamburg, Germany. describe deep-inelastic lepton scattering from the deuteron $[12-14]$ and the three-nucleon bound state simultaneously.

Deep-inelastic lepton scattering from nuclei is described in front form dynamics. Front form dynamics allows the use of impulse approximation consistently [15-17]. This is a formal quantum mechanical consistency. We believe that impulse approximation is also physically reliable for the description of deep-inelastic lepton scattering. The form of plane wave impulse approximation (PWIA) used neglects the interaction between the hadrons, produced on the struck nucleon, and the spectator residual nucleus in the final state, and it neglects all many-hadron currents. The paper extends the successful description, given previously [18] for deepinelastic scattering of unpolarized leptons from unpolarized

${ }^{3} \mathrm{He}$, to the case with polarization. Section II recalls some facts on front form dynamics needed for the actual calculation. Section III parametrizes the nuclear current tensor of a spin- $\frac{1}{2}$ target; it introduces the spin-averaged and the spindependent structure functions; the generalization to spin-1 targets, e.g., to the deuteron, is given in the Appendixes A and B. Section IV describes the cross section for longitudinal lepton polarization in terms of the nuclear structure functions. Section V derives convolution formulas for the spindependent structure functions in PWIA. They require the spin distributions of the deuteron and the trinucleon bound states in terms of front form variables; those front form spin distributions are determined in Sec. VI for the trinucleon bound state and in Appendix $\mathrm{C}$ for the deuteron. Our results are discussed in Sec. VII. Conclusions are given in Sec. VIII.

\section{ELEMENTS OF FRONT FORM DYNAMICS}

First, we give our notation. We exhibit the instant form components of any four-vector $A$ in round brackets as usual, i.e.,

$$
A=\left(A^{0}, A^{1}, A^{2}, A^{3}\right)
$$

and abbreviate the three spatial components $\left(A^{1}, A^{2}, A^{3}\right)$ by $\vec{A}$. We use the metric $g^{\mu \nu}=(1,-1,-1,-1)$ and the four-

(C) 1997 The American Physical Society 
dimensional totally antisymmetric tensor $\epsilon^{\mu \nu \alpha \beta}$ with $\epsilon_{0123}=-\epsilon^{0123}=1$. For the description of front form dynamics we choose the following basis vectors:

$$
\begin{gathered}
n=(1,-\hat{n}), \\
l=(1, \hat{n}), \\
e_{1}=\left(0, \hat{e}_{1}\right), \\
e_{2}=\left(0, \hat{e}_{2}\right) .
\end{gathered}
$$

They satisfy the conditions $n^{2}=l^{2}=0, e_{i} \cdot e_{j}=-\delta_{i j}$, $\frac{1}{2} n \cdot l=1$ and $n \cdot e_{i}=l \cdot e_{i}=0$. The vectors $n$ and $l$ are null vectors. The three-dimensional vectors $\hat{e}_{1}, \hat{e}_{2}$, and $\hat{n}$ form a positively oriented three-dimensional set of orthonormal basis vectors such that

$$
\frac{1}{2} e_{1 \mu} e_{2 \nu} \epsilon^{\mu \nu \alpha \beta} n_{\alpha} l_{\beta}=1
$$

the carets over vector symbols indicate three-dimensional unit vectors. Four-dimensional vectors are built up from those basis vectors, i.e.,

$$
A=\frac{1}{2} A^{-} n+\frac{1}{2} A^{+} l+A^{1} e_{1}+A^{2} e_{2},
$$

where the front form components

$$
\begin{gathered}
A^{-}=l \cdot A=A^{0}-\hat{n} \cdot \vec{A}, \\
A^{+}=n \cdot A=A^{0}+\hat{n} \cdot \vec{A}, \\
A^{1}=-e_{1} \cdot A, \\
A^{2}=-e_{2} \cdot A
\end{gathered}
$$

are introduced. We arrange the front form components of a vector in curly brackets, i.e.,

$$
\begin{gathered}
A=\left\{A^{-}, A^{1}, A^{2}, A^{+}\right\}, \\
A=\left\{A^{-}, \mathbf{A}\right\},
\end{gathered}
$$

and abbreviate the three kinematic components $\left\{A^{1}, A^{2}, A^{+}\right\}$by $\mathbf{A}$ and the transverse components $\left\{A^{1}, A^{2}\right\}$ among them by $\mathbf{A}_{\perp}$. The three-dimensional basis vectors $\hat{e}_{1}, \hat{e}_{2}$, and $\hat{n}$ of front form dynamics may be assumed to differ from those of instant form dynamics; thus, the components $\left\{A^{1}, A^{2}\right\}$ and $\left(A^{1}, A^{2}\right)$ may be different; in this paper we choose them to be identical. The four-dimensional volume element of integration is

$$
\begin{gathered}
d^{4} A=d A^{0} d A^{1} d A^{2} d A^{3}, \\
d^{4} A=\frac{1}{2} d A^{-} d A^{1} d A^{2} d A^{+},
\end{gathered}
$$

we shall use the abbreviations $d^{3} \vec{A}=d A^{1} d A^{2} d A^{3}$ and $d^{3} \mathbf{A}=d A^{1} d A^{2} d A^{+}=d \mathbf{A}_{\perp} d A^{+}$.
In the description of deep-inelastic lepton scattering the front form null vectors $n$ and $l$ are chosen by

$$
\hat{n}=-\left(\frac{Q^{0}}{\sqrt{\left(Q^{0}\right)^{2}-Q^{2}}} \hat{Q}+\sqrt{\frac{-Q^{2}}{\left(Q^{0}\right)^{2}-Q^{2}}} \frac{\hat{k}_{e}-\left(\hat{k}_{e} \cdot \hat{Q}\right) \hat{Q}}{\sqrt{1-\left(\hat{k}_{e} \cdot \hat{Q}\right)^{2}}}\right),
$$

$k_{e}$ being the initial four-momentum of the lepton and $Q$ its momentum transfer to the target nucleus. The choice (2.7) yields $Q^{+}=Q^{0}+\hat{n} \cdot \vec{Q}=0$. The basis vector $e_{1}$ is defined by

$$
\hat{e}_{1}=\frac{\mathbf{Q}_{\perp}}{\left|\mathbf{Q}_{\perp}\right|}
$$

the definition of $e_{2}$ follows then from the requirement (2.3). Thus, the momentum transfer $Q$ has the representation

$$
Q=\left\{2 Q^{0}, \sqrt{-Q^{2}}, 0,0\right\}
$$

Second, we need the description of states for the nuclear target and for the constituents of the nuclear target. For the description we use eigenstates of the mass operator $M$, of the front form components $\mathbf{P}$ of the four-momentum $P$ and of the spin $\vec{J}_{f}$. The spin operator $\vec{J}_{f}$ is defined in terms of the Pauli-Lubanski vector $W[16]$, i.e.,

$$
\left(0, \vec{J}_{f}\right)^{\mu}=\frac{1}{M} L_{f}^{-1}\left(\frac{P}{M}\right)_{\nu}^{\mu} W^{\nu}
$$

The Lorentz transformation $L_{f}(P / M)$ boosts a particle of mass $M$ from rest to four-momentum $P$. In Eq. (2.10) the Lorentz transformation $L_{f}(P / M)$ is still operator valued in its dependence on mass $M$ and momentum $P$. The corresponding boosts $\Lambda_{f}(P / M)$ belong to the front form kinematic subgroup of the Lorentz group; thus, even for composite systems with interactions between constituents, the boosts $\Lambda_{f}(P / M)$ are interaction free; this is why the components $\mathbf{P}$ of the momentum are called kinematic. The eigenstates are chosen in the form

$$
\left(\begin{array}{c}
M^{2} \\
\mathbf{P} \\
-W^{2} / M^{2} \\
-v \cdot W / M
\end{array}\right)|\mathbf{p} \lambda\rangle=\left(\begin{array}{c}
m^{2} \\
\mathbf{p} \\
j(j+1) \\
\lambda
\end{array}\right)|\mathbf{p} \lambda\rangle .
$$

The defining spin operators are given covariantly, e.g., $-W^{2} / M^{2}=\vec{J}_{f}^{2}$. The spin projection $-v \cdot W / M$ is chosen with respect to the direction of quantization $v, v$ taken as a spacelike vector which satisfies $v^{2}=-1$ and $v \cdot p=0$. The standard choice of $v$ for front form dynamics is

$$
v=\frac{p}{m}-\frac{m}{n \cdot p} n \text {. }
$$

In this case the spin projection operator becomes $-v \cdot W / M=\hat{n} \cdot \vec{J}_{f}$. For a spin- $\frac{1}{2}$ particle the operator $\vec{J}_{f}$ is realized by the vector of Pauli matrices $\vec{\sigma} / 2$. The notation of states does not keep the eigenvalues of mass and spin, $m$ and $j$; it only indicates the kinematic components of the momen- 
tum and the spin projection, $\mathbf{p}$ and $\lambda$. The states (2.11) are orthonormal and complete, i.e.,

$$
\begin{gathered}
\left\langle\mathbf{p}^{\prime} \lambda^{\prime} \mid \mathbf{p} \lambda\right\rangle=\delta\left(\mathbf{p}^{\prime}-\mathbf{p}\right) \delta_{\lambda^{\prime} \lambda}, \\
\sum_{\lambda} \int d^{3} \mathbf{p}|\mathbf{p} \lambda\rangle\langle\mathbf{p} \lambda|=I .
\end{gathered}
$$

They are connected to the states $\left|\mathbf{p}_{R} \lambda\right\rangle$ in the particles rest frame with $p_{R}=\{m, 0,0, m\}$ by the unitary transformation $U\left[\Lambda_{f}(p / m)\right]$ according to

$$
|\mathbf{p} \lambda\rangle=\sqrt{\frac{m}{p^{+}}} U\left[\Lambda_{f}\left(\frac{p}{m}\right)\right]\left|\mathbf{p}_{R} \lambda\right\rangle,
$$

the extra factor $\sqrt{m / p^{+}}$ensuring orthogonality in the form (2.13a). The transformation does not change the eigenvalue $\lambda$.

Need will arise to also use basis states $|\vec{p} s\rangle$ in the quantum mechanical framework of instant form dynamics. The latter states are defined in the same way as the corresponding front form states $|\mathbf{p} \lambda\rangle$ are in Eq. (2.11). Differences are due to the different choice of kinematic components of the momentum. Though the components $\vec{p}$ are called kinematic, the instant form boosts $\Lambda_{c}(p / m)$ are not interactionfree for composite systems, in contrast to the properties of the front form boosts $\Lambda_{f}(p / m)$; the indices $c$ and $f$ differentiate the form of dynamics, $c$ standing for canonical represents the instant form, $f$ standing for front represents the front form of dynamics. In addition, the instant form boosts $\Lambda_{c}(p / m)$ of a particle with mass $m$ from rest to the momentum $p$ are rotationless, whereas the corresponding front form boosts $\Lambda_{f}(p / m)$ contain a rotation. Thus, the same four-vector $W$ corresponds to different rest frame vectors $\vec{J}_{c}$ in instant and $\vec{J}_{f}$ in front form dynamics. They are related [16] by the Melosh rotation $R_{M}(p)$, i.e.,

$$
\begin{gathered}
R_{M}(p)=\Lambda_{f}^{-1}\left(\frac{p}{m}\right) \Lambda_{c}\left(\frac{p}{m}\right), \\
J_{c}^{i}=\sum_{j} R_{M}(p)^{i} J_{j}^{j} .
\end{gathered}
$$

The Melosh rotation is an operator in spin space. For a spin$\frac{1}{2}$ particle it takes the explicit form

$$
R_{M}(p)=\frac{m+p^{+}+i \hat{n} \cdot(\vec{\sigma} \times \vec{p})}{\sqrt{\left(m+p^{+}\right)^{2}+\mathbf{p}_{\perp}^{2}}} .
$$

The quantum mechanical states are related to each other by

$$
|\vec{p} s\rangle=\sum_{\lambda}|\mathbf{p} \lambda\rangle \sqrt{\left|\frac{\partial \mathbf{p}}{\partial \vec{p}}\right|}\left\langle\lambda\left|R_{M}^{+}(p)\right| s\right\rangle .
$$

In Eq. (2.16) $|\partial \mathbf{p} / \partial \vec{p}|=p^{+} / p^{0}$ is the Jacobian for the transformation of momentum variables. The instant form states $|\vec{p} s\rangle$ are also chosen to be orthogonal and complete without extra weight factors in their phase space as the front form states are chosen according to Eqs. (2.13a) and (2.13b).

\section{NUCLEAR CURRENT TENSOR}

The current tensor $\left\langle n_{A}\left|W_{A}^{\mu \nu}\left(Q, P_{A}\right)\right| n_{A}\right\rangle$ of the target $A$ with mass $m_{A}$, four-momentum $P_{A}$, and polarization $n_{A}$ is required for the description of inclusive processes; it is defined by

$$
\begin{aligned}
\left\langle n_{A}\left|W_{A}^{\mu \nu}\left(Q, P_{A}\right)\right| n_{A}\right\rangle= & (2 \pi)^{6} \sum_{\beta_{X}} \int d^{3} \mathbf{P}_{X} \sqrt{\frac{P_{A}^{+}}{m_{A}}}\left\langle\mathbf{P}_{A} n_{A}\left|J_{A}^{\mu}(0)\right| \mathbf{P}_{X} \beta_{X}\right\rangle 2 \delta^{3}\left(\mathbf{P}_{X}-\mathbf{Q}-\mathbf{P}_{A}\right) \delta\left(P_{X}^{-}-Q^{-}-P_{A}^{-}\right) \\
& \times\left\langle\mathbf{P}_{X} \beta_{X}\left|J_{A}^{\nu}(0)\right| \mathbf{P}_{A} n_{A}\right\rangle \sqrt{\frac{P_{A}^{+}}{m_{A}}}
\end{aligned}
$$

in terms of the nuclear current $J_{A}^{\mu}(0)$ at time-space point 0 ; the proton charge $e_{p}$ is split off from the current. General final states $\left|\mathbf{P}_{X} \beta_{X}\right\rangle$ of c.m. momentum $\mathbf{P}_{X}$ can be reached in the scattering process, $\beta_{X}$ describing discrete quantum numbers and also the modes of internal excitation; they are on mass shell, they are not observed in inclusive processes. The momentum transfer to the target nucleus is $Q$. The current tensor $\left\langle n_{A}\left|W_{A}^{\mu \nu}\left(Q, P_{A}\right)\right| n_{A}\right\rangle$ is obtained from its spinoperator form $W_{A}^{\mu \nu}\left(Q, P_{A}\right)$ and from the density operator $\rho_{A}\left(n_{A} P_{A}\right)$ by

$$
\left\langle n_{A}\left|W_{A}^{\mu \nu}\left(Q, P_{A}\right)\right| n_{A}\right\rangle=\operatorname{Tr}\left[W_{A}^{\mu \nu}\left(Q, P_{A}\right) \rho_{A}\left(n_{A} P_{A}\right)\right] .
$$

Deuteron and ${ }^{3} \mathrm{He}$ are the considered target nuclei. They have spin 1 and $\frac{1}{2}$, respectively; for calculations they are assumed to be in pure quantum mechanical states $\left|\mathbf{P}_{A} n_{A}\right\rangle$. They are defined as eigenstates of the mass $M$, the momentum $\mathbf{P}$, the spin $-W^{2} / M^{2}$, and of the spin projection $-n_{A} \cdot W / M$ in the direction of polarization $n_{A}$ with eigenvalue $j$, i.e., 1 and $\frac{1}{2}$, respectively. Unless stated otherwise, the plane-wave $\delta$-function producing part of the states $\left|\mathbf{P}_{A} n_{A}\right\rangle$ will be considered to be split off in the following. Though the target states are assumed to be quantum mechanically pure for the purpose of calculation, polarization will nevertheless be described by the density operator $\rho_{A}\left(n_{A} P_{A}\right)$. It is an operator in nuclear spin space. It is a Lorentz scalar and normalized by $\operatorname{Tr}\left[\rho_{A}\left(n_{A} P_{A}\right)\right]=1$. When referring to pure states as assumed, it has the property $\rho_{A}^{2}\left(n_{A} P_{A}\right)=\rho_{A}\left(n_{A} P_{A}\right)$. The polarization $n_{A}$ chosen by experimentalists does in general not coincide with the standard 
direction $v$ of quantization in Eq. (2.12). Thus, the target states $\left|\mathbf{P}_{A} n_{A}\right\rangle$ are to be expanded in terms of the target basis states $\left|\mathbf{P}_{A} \lambda_{A}\right\rangle$ of Eq. (2.11), i.e.,

$$
\left|\mathbf{P}_{A} n_{A}\right\rangle=\sum_{\lambda_{A}}\left|\mathbf{P}_{A} \lambda_{A}\right\rangle\left\langle\lambda_{A} \mid n_{A}\right\rangle
$$

the transformation parameters $\left\langle\lambda_{A} \mid n_{A}\right\rangle$ depend on the momentum $P_{A}$; the trace operation in Eq. (3.2) is carried out with respect to matrix elements of the basis states $\left|\mathbf{P}_{A} \lambda_{A}\right\rangle$ at fixed target momentum $\mathbf{P}_{A}$.

\section{A. Trinucleon density operator}

The full density operator $\rho_{A}\left(n_{A}\right)$ commutes with the momentum operator $P$. Its projection $\rho_{A}\left(n_{A} P_{A}\right)$, i.e.,

$$
\left\langle\mathbf{P}_{A} \lambda_{A}^{\prime}\left|\rho_{A}\left(n_{A}\right)\right| \mathbf{P}_{A} \lambda_{A}\right\rangle=\left\langle\lambda_{A}^{\prime}\left|\rho_{A}\left(n_{A} P_{A}\right)\right| \lambda_{A}\right\rangle,
$$

onto the Hilbert sector of momentum eigenstates with eigenvalue $\mathbf{P}_{A}$ is needed in Eq. (3.2). The density operator of a spin- $\frac{1}{2}$ target is parametrized in the form

$$
\rho_{A}\left(n_{A} P_{A}\right)=\frac{1}{2}\left[1-\frac{2}{m_{A}} n_{A} \cdot W\right] .
$$

The parametrization satisfies all required constraints. It is at most linear in the Pauli-Lubanski vector $W$ which is traceless, i.e., $\operatorname{Tr}[W]=0$. The vector $n_{A}$ is the polarization vector according to which target polarization is defined. The parametrization (3.5) satisfies all required constraints for a quantum mechanically pure target state, provided $n_{A}^{2}=-1$ and $n_{A} \cdot P_{A}=0$.

Matrix elements of the density operator of any momentum $P_{A}$ are related to those of the rest frame, i.e.,

$$
\left\langle\lambda_{A}^{\prime}\left|\rho_{A}\left(n_{A} P_{A}\right)\right| \lambda_{A}\right\rangle=\left\langle\lambda_{A}^{\prime}\left|\frac{1}{2}\left[1+\hat{n}_{R} \cdot \vec{\sigma}_{A}\right]\right| \lambda_{A}\right\rangle .
$$

For Eq. (3.6) the identities

$$
\begin{aligned}
\frac{W^{\mu}}{m_{A}}\left|\mathbf{P}_{A} \lambda_{A}\right\rangle= & \sqrt{\frac{m_{A}}{P_{A}^{+}}} U\left[\Lambda_{f}\left(\frac{P_{A}}{m_{A}}\right)\right]\left[L_{f}\left(\frac{P_{A}}{m_{A}}\right)_{\nu}^{\mu}\left(0, \frac{\vec{\sigma}_{A}}{2}\right)^{\nu}\right] \\
& \times\left|\mathbf{P}_{R} \lambda_{A}\right\rangle \\
& n_{A}^{\mu}=L_{f}\left(\frac{P_{A}}{m_{A}}\right)_{\nu}^{\mu}\left(0, \hat{n}_{R}\right)^{\nu}
\end{aligned}
$$

are used, $P_{R}=\left\{m_{A}, 0,0, m_{A}\right\}$ denoting the target rest frame momentum, $n_{R}=\left(0, \hat{n}_{R}\right)$ its rest frame polarization and $\vec{\sigma}_{A} / 2$ its angular momentum operator. The constraints on $\rho_{A}\left(n_{A} P_{A}\right)$ are best proven in the rest frame.

The corresponding density operator of a spin-1 target is given in Appendix A.

\section{B. Trinucleon current tensor operator}

The current tensor operator $W_{A}^{\mu \nu}\left(Q, P_{A}\right)$ is Hermitian and conserved, it preserves parity and time-reversal invariance.
Its Lorentz structure is built from the three four-vectors $Q$, $P_{A}$, and $W$. The current tensor operator $W_{A}^{\mu \nu}\left(Q, P_{A}\right)$ has the general form

$$
\begin{aligned}
W_{A}^{\mu \nu}\left(Q, P_{A}\right)= & {\left[\frac{Q^{\mu} Q^{\nu}}{Q^{2}}-g^{\mu \nu}\right] F_{1}^{A}\left(x, Q^{2}\right) \frac{A}{m_{A}} } \\
& +\frac{\widetilde{P_{A}^{\mu}} \widetilde{P}_{A}^{\nu}}{Q \cdot P_{A}} F_{2}^{A}\left(x, Q^{2}\right) \frac{1}{m_{A}} \\
& +i \frac{1}{j} \frac{\epsilon^{\mu \nu \alpha \beta}}{Q \cdot P_{A}} Q_{\alpha}\left[W_{\beta} g_{1}^{A}\left(x, Q^{2}\right)\right. \\
& \left.+\left(W_{\beta}-\frac{Q \cdot W}{Q \cdot P_{A}} P_{A \beta}\right) g_{2}^{A}\left(x, Q^{2}\right)\right] \frac{A}{m_{A}}
\end{aligned}
$$

with

$$
\begin{gathered}
\widetilde{P}_{A}^{\mu}:=P_{A}^{\mu}-\frac{Q \cdot P_{A}}{Q^{2}} Q^{\mu}, \\
x:=-\frac{A Q^{2}}{2 Q \cdot P_{A}},
\end{gathered}
$$

$x$ being the Bjorken scaling variable. The dependence of the current tensor operator on the Pauli-Lubanski vector $W$ is linear for a spin- $\frac{1}{2}$ target, $j=\frac{1}{2}$ being the spin quantum number. The current tensor operator is built from four basic tensor forms which satisfy all symmetry requirements. Those basic tensor forms get augmented by structure functions which are real-valued Lorentz scalars and therefore depend on the nontrivial scalars $Q^{2}$ and $Q \cdot P_{A}$ or, equivalently, on the Bjorken scaling variable $x$ and $Q^{2}$.

The current tensor $\left\langle n_{A}\left|W_{A}^{\mu \nu}\left(Q, P_{A}\right)\right| n_{A}\right\rangle$ follows from the current tensor operator $W_{A}^{\mu \nu}\left(Q, P_{A}\right)$ of Eq. (3.8) and from the density operator $\rho_{A}\left(n_{A} P_{A}\right)$ of Eq. (3.5) according to Eq. (3.2). In fact, the current tensor $\left\langle n_{A}\left|W_{A}^{\mu \nu}\left(Q, P_{A}\right)\right| n_{A}\right\rangle$ is obtained from the operator (3.8) by replacing the PauliLubanski vector $W$ by the polarization vector $n_{A}$ according to the relation

$$
n_{A}^{\mu}=\left(1 / j m_{A}\right) \operatorname{Tr}\left[W^{\mu} \rho_{A}\left(n_{A}, P_{A}\right)\right] .
$$

The two spin-averaged structure functions $F_{1}^{A}$ and $F_{2}^{A}$ and the two spin-dependent structure functions $g_{1}^{A}$ and $g_{2}^{A}$ determine the current tensor in full; they are used in the notation standard for deep-inelastic lepton scattering; they are related to the corresponding structure functions $W_{1}^{A}, W_{2}^{A}, G_{1}^{A}$, and $G_{2}^{A}$ of Ref. [3], usually employed at lower energy and momentum transfers, by

$$
F_{1}^{A}\left(x, Q^{2}\right)=\frac{m_{A}}{A} W_{1}^{A}\left(Q^{2}, Q \cdot P_{A} / m_{A}\right)
$$

$$
F_{2}^{A}\left(x, Q^{2}\right)=\frac{Q \cdot P_{A}}{m_{A}} W_{2}^{A}\left(Q^{2}, Q \cdot P_{A} / m_{A}\right),
$$




$$
\begin{gathered}
g_{1}^{A}\left(x, Q^{2}\right)=\frac{Q \cdot P_{A}}{A m_{A}} G_{1}^{A}\left(Q^{2}, Q \cdot P_{A} / m_{A}\right), \\
g_{2}^{A}\left(x, Q^{2}\right)=\frac{\left(Q \cdot P_{A}\right)^{2}}{A m_{A}^{3}} G_{2}^{A}\left(Q^{2}, Q \cdot P_{A} / m_{A}\right)
\end{gathered}
$$

We are interested in describing asymmetry measurements in deep-inelastic lepton scattering; they are carried out in order to determine the spin-structure functions $g_{1}^{A}$ and $g_{2}^{A}$.

All structure functions can be obtained from a given current tensor by contractions with other tensors. The deuteron current tensor is - as current tensor of a spin-1 target-quite different from Eq. (3.8) as Appendix B shows. Nevertheless, its dependence on the spin-structure functions $g_{1}^{A}$ and $g_{2}^{A}$, being contained in the part proportional to $\epsilon^{\mu \nu \alpha \beta}$, is the same also for the deuteron. Thus, the relations by which the spin structure functions $g_{1}^{A}$ and $g_{2}^{A}$ are recovered from a computed current tensor will hold for the deuteron in identical form.

\section{Trinucleon current tensor in the nuclear c.m. system}

The nuclear current tensor $W_{A}^{\mu \nu}\left(Q, P_{A}\right)$ is evaluated in the nuclear c.m. system. We shall now use the notational identifications $P_{A}=P_{R}$ for the four-momentum of the target and $n_{A}=n_{R}$ for its polarization, i.e., $P_{A}=\left\{m_{A}, 0,0, m_{A}\right\}=\left(m_{A}, \overrightarrow{0}\right)$ and $n_{A}=\left\{-\left(n \cdot n_{A}\right), n_{A}^{1}, n_{A}^{2},\left(n \cdot n_{A}\right)\right\}=\left(0, \hat{n}_{R}\right)$. The momentum transfer is used in the form (2.9). The kinematically needed four-vectors can be given in terms of the basis vectors $n, l, e_{1}$, and $e_{2}$, i.e.,

$$
\begin{gathered}
P_{A}=\frac{1}{2} m_{A}(n+l), \\
Q=\frac{1}{2} Q^{-} n+\sqrt{-Q^{2}} e_{1}, \\
n_{A}=\frac{1}{2}\left(n \cdot n_{A}\right)(n-l)-\left(e_{1} \cdot n_{A}\right) e_{1}-\left(e_{2} \cdot n_{A}\right) e_{2} \\
n_{A}-\frac{Q \cdot n_{A}}{Q \cdot P_{A}} P_{A}=-\left(\left(n \cdot n_{A}\right)+\frac{\sqrt{-Q^{2}}}{Q^{-}}\left(e_{1} \cdot n_{A}\right)\right) n \\
-\frac{\sqrt{-Q^{2}}}{Q^{-}}\left(e_{1} \cdot n_{A}\right) l-\left(e_{1} \cdot n_{A}\right) e_{1} \\
-\left(e_{2} \cdot n_{A}\right) e_{2} \cdot
\end{gathered}
$$

The nuclear current tensor takes the form

$$
\begin{aligned}
\left\langle n_{A}\left|W_{A}^{\mu \nu}\left(Q, P_{A}\right)\right| n_{A}\right\rangle= & {\left[\frac{Q^{\mu} Q^{\nu}}{Q^{2}}-g^{\mu \nu}\right] F_{1}^{A}\left(x, Q^{2}\right) \frac{A}{m_{A}}+\frac{\widetilde{P}_{A}^{\mu} \widetilde{P}_{A}^{\nu}}{Q \cdot P_{A}} F_{2}^{A}\left(x, Q^{2}\right) \frac{1}{m_{A}}+\frac{i A}{m_{A}} \epsilon^{\mu \nu \alpha \beta}\left(n_{\alpha}+\frac{2 \sqrt{-Q^{2}}}{Q^{-}} e_{1 \alpha}\right) } \\
& \times\left\{-n_{\beta}\left[\frac{1}{2}\left(n \cdot n_{A}\right) g_{1}^{A}\left(x, Q^{2}\right)+\left(\left(n \cdot n_{A}\right)+\frac{\sqrt{-Q^{2}}}{Q^{-}}\left(e_{1} \cdot n_{A}\right)\right) g_{2}^{A}\left(x, Q^{2}\right)\right]+l_{\beta}\left(\frac{1}{2}\left(n \cdot n_{A}\right) g_{1}^{A}\left(x, Q^{2}\right)\right.\right. \\
& \left.-\frac{\sqrt{-Q^{2}}}{Q^{-}}\left(e_{1} \cdot n_{A}\right) g_{2}^{A}\left(x, Q^{2}\right)\right)-e_{1 \beta}\left(e_{1} \cdot n_{A}\right)\left[g_{1}^{A}\left(x, Q^{2}\right)+g_{2}^{A}\left(x, Q^{2}\right)\right] \\
& \left.-e_{2 \beta}\left(e_{2} \cdot n_{A}\right)\left[g_{1}^{A}\left(x, Q^{2}\right)+g_{2}^{A}\left(x, Q^{2}\right)\right]\right\} .
\end{aligned}
$$

Equation (3.13) rewrites Eq. (3.8) — after the trace operation (3.2) is carried out — with respect to the antisymmetric part of the nuclear current tensor; only that part matters for the extraction of the spin-structure functions $g_{1}^{A}$ and $g_{2}^{A}$; it is the same for the current tensors of the three-nucleon bound states and of the deuteron.

\section{Extraction of spin-structure functions}

The spin-structure functions $g_{1}^{A}$ and $g_{2}^{A}$ can be extracted from particular matrix elements or by contractions with fourvectors and tensors available from the kinematic setup of the experiment as in Ref. [3]. We label the first extraction scheme by I, the second extraction scheme by II.

The extraction scheme I reads the nuclear spin-structure functions $g_{1}^{A}$ and $g_{2}^{A}$ off from selected components of the nuclear current tensor. The considered matrix element is written covariantly as a contraction with the basis vectors $e_{1}$ and $e_{2}$, i.e.,

$$
e_{1 \mu} e_{2 \nu}\left\langle n_{A}\left|W_{A}^{\mu \nu}\left(Q, P_{A}\right)\right| n_{A}\right\rangle=-\frac{i A}{m_{A}}\left(\left(n \cdot n_{A}\right) g_{1}^{A}\left(x, Q^{2}\right)+2 \frac{\sqrt{-Q^{2}}}{Q^{-}}\left(e_{1} \cdot n_{A}\right) g_{2}^{A}\left(x, Q^{2}\right)\right) \text {, }
$$

it refers to the kinematic components of the current. When specifying the nuclear polarization $n_{A}$ in the target rest frame, both nuclear spin-structure functions can be extracted separately from that single relation, i.e.,

$$
g_{1}^{A}\left(x, Q^{2}\right)=-\left.\left(i m_{A} / A\right) e_{1 \mu} e_{2 \nu}\left\langle n_{A}\left|W_{A}^{\mu \nu}\left(P_{A}, Q\right)\right| n_{A}\right\rangle\right|_{n_{A}} \| \hat{n},
$$




$$
g_{2}^{A}\left(x, Q^{2}\right)=-\left(i m_{A} / A\right) \frac{Q^{-}}{2 \sqrt{-Q^{2}}} e_{1 \mu} e_{2 \nu}\left\langle n_{A}\left|W_{A}^{\mu \nu}\left(P_{A}, Q\right)\right| n_{A}\right\rangle \mid \vec{n}_{A} \| \hat{e}_{1} .
$$

The extraction scheme II contracts the full current tensor with the tensors of other physical variables and extracts the spin-structure functions from the resulting scalars, i.e.,

$$
\begin{gathered}
g_{1}^{A}\left(x, Q^{2}\right)=\frac{-i}{2 A} \frac{Q \cdot P_{A}}{Q \cdot n_{A}} \frac{Q \cdot n_{A} P_{A}^{2} Q^{\alpha} n_{A}^{\beta}-Q \cdot P_{A} Q^{\alpha} P_{A}^{\beta}}{P_{A}^{2}\left[Q^{2}+\left(Q \cdot n_{A}\right)^{2}\right]-\left(Q \cdot P_{A}\right)^{2}} \epsilon_{\alpha \beta \mu \nu}\left\langle n_{A}\left|W_{A}^{\mu \nu}\left(Q, P_{A}\right)\right| n_{A}\right\rangle, \\
g_{2}^{A}\left(x, Q^{2}\right)=\frac{i}{2 A} \frac{\left(Q \cdot P_{A}\right)^{2}}{Q^{2} Q \cdot n_{A}} \frac{Q \cdot n_{A} Q \cdot P_{A} Q^{\alpha} n_{A}^{\beta}-\left[Q^{2}+\left(Q \cdot n_{A}\right)^{2}\right] Q^{\alpha} P_{A}^{\beta}}{P_{A}^{2}\left[Q^{2}+\left(Q \cdot n_{A}\right)^{2}\right]-\left(Q \cdot P_{A}\right)^{2}} \epsilon_{\alpha \beta \mu \nu}\left\langle n_{A}\left|W_{A}^{\mu \nu}\left(Q, P_{A}\right)\right| n_{A}\right\rangle .
\end{gathered}
$$

The extraction scheme II uses relations (3.11c) and (3.11d) for the spin-structure functions $G_{i}^{A}$ of Ref. [3]. Both extraction schemes-and all possible variants of them-are equivalent as long as the current tensor is exact, i.e., satisfies Lorentz covariance and current conservation. However, they are inequivalent for approximate current tensors. And the nuclear current tensor of this paper will only be calculated approximately in PWIA and with wave functions which do not exhibit all required symmetry properties as Sec. VI will explain in more detail.

\section{CROSS SECTION}

The derivation of the inclusive cross section for inelastic lepton scattering is recalled. Let $k_{e}=\left(E, \vec{k}_{e}\right)$ and $k_{e}^{\prime}=\left(E^{\prime}, \vec{\Omega}_{e}^{\prime} E^{\prime}\right)$ be the initial and final momenta of the lepton and $Q=k_{e}-k_{e}^{\prime}$ the four-momentum transfer. The cross section is determined by the nuclear current tensor $\left\langle n_{A}\left|W_{A}^{\mu \nu}\left(Q, P_{A}\right)\right| n_{A}\right\rangle$ of Sec. III and by the corresponding lepton current tensor $\left\langle n_{e}\left|\eta_{\mu \nu}\right| n_{e}\right\rangle$ with

$$
\begin{aligned}
\left\langle n_{e}\left|\eta^{\mu \nu}\right| n_{e}\right\rangle= & 2\left(k_{e}^{\prime \mu} k_{e}^{\nu}+k_{e}^{\prime \nu} k_{e}^{\mu}-g^{\mu \nu} k_{e} \cdot k_{e}^{\prime}\right. \\
& \left.+i m_{e} \epsilon^{\mu \nu \alpha \beta} Q_{\alpha} n_{e \beta}\right) .
\end{aligned}
$$

When the lepton is longitudinally polarized before scattering, its polarization vector $n_{e}$ gets

$$
n_{e}=h_{e} \frac{k_{e}}{m_{e}}
$$

with $h_{e}= \pm 1$ being the helicity and $m_{e}$ the mass of the lepton. In the rest frame of the target the cross section becomes

$$
\frac{d^{2} \sigma\left(h_{e}\right)}{d \Omega_{e}^{\prime} d E^{\prime}}=\frac{\alpha^{2}}{Q^{4}} \frac{E^{\prime}}{E}\left\langle n_{e}\left|\eta_{\mu \nu}\right| n_{e}\right\rangle\left\langle n_{A}\left|W_{A}^{\mu \nu}\left(Q, P_{A}\right)\right| n_{A}\right\rangle .
$$

It is split into a helicity-independent and a helicity-dependent part, i.e.,

$$
\frac{d^{2} \sigma\left(h_{e}\right)}{d \Omega_{e}^{\prime} d E^{\prime}}=\frac{d^{2} \sigma(\text { unpol })}{d \Omega_{e}^{\prime} d E^{\prime}}+h_{e} \frac{d^{2} \sigma(\mathrm{pol})}{d \Omega_{e}^{\prime} d E^{\prime}} .
$$

Its polarization part has the form

$$
\begin{gathered}
\frac{d^{2} \sigma(p o l)}{d \Omega_{e}^{\prime} d E^{\prime}}=\frac{1}{2}\left(\frac{d^{2} \sigma(+1)}{d \Omega_{e}^{\prime} d E^{\prime}}-\frac{d^{2} \sigma(-1)}{d \Omega_{e}^{\prime} d E^{\prime}}\right) \\
\frac{d^{2} \sigma(p o l)}{d \Omega_{e}^{\prime} d E^{\prime}}= \\
\sigma_{\mathrm{Mott}^{2}} \tan ^{2} \frac{\Theta_{e}}{2}\left\{\left[E \cos \theta_{A}+E^{\prime}\left(\cos \Theta_{e} \cos \theta_{A}\right.\right.\right. \\
\left.\left.+\sin \Theta_{e} \sin \theta_{A} \cos \phi_{A}\right)\right] \frac{g_{1}^{A}\left(x, Q^{2}\right)}{m_{A} Q^{0}} \\
-2 E E^{\prime}\left[\cos \theta_{A}-\left(\cos \Theta_{e} \cos \theta_{A}\right.\right. \\
\left.\left.\left.+\sin \Theta_{e} \sin \theta_{A} \cos \phi_{A}\right)\right] \frac{g_{2}^{A}\left(x, Q^{2}\right)}{m_{A}\left(Q^{0}\right)^{2}}\right\}
\end{gathered}
$$

It is the same for spin- $\frac{1}{2}$ and the spin- 1 targets; $\Theta_{e}$ is the scattering angle of the lepton, $\sigma_{\text {Mott }}$ the Mott cross section. In both cases the target polarization $n_{A}$ is described in the rest frame by the polarization angles $\theta_{A}$ and $\phi_{A}$, i.e., $n_{A}=\left(0, \sin \theta_{A} \cos \phi_{A}, \sin \theta_{A} \sin \phi_{A}, \cos \theta_{A}\right)$. The angles are measured with respect to the incoming lepton beam.

\section{PLANE WAVE IMPULSE APPROXIMATION}

The calculations of this paper are carried out in PWIA. They extend the work of Ref. [18] by the inclusion of beam and target polarization. Reference [18] also uses PWIA. Superficially, impulse approximation assumes that only onenucleon currents $j_{N(i)}^{\mu}(0)$ contribute to the complete nuclear current $J_{A}^{\mu}(0)$, i.e.,

$$
J_{A}^{\mu}(0)=\sum_{i=1}^{A} j_{N(i)}^{\mu}
$$

Stated in this manner, the assumption is clearly inconsistent because, for interacting systems, the current on the righthand side does not transform correctly as a four-vector. In contrast, the restricted assumption

$$
\mathbf{J}_{A}(0)=\sum_{i=1}^{A} \mathbf{j}_{N(i)}
$$

is consistent: Both sides of the latter equation transform correctly under the front form kinematic subgroup of the Lor- 
entz group; the full nuclear current can then be generated by translations and general Lorentz transformations. Furthermore, inclusive cross sections require knowledge of the nuclear current tensor which is quadratic in the current operator and which therefore contains interference terms of the form $j_{N(i)}^{\mu} j_{N(k)}^{\nu}$. PWIA neglects those interference terms. Reference [3] discusses this approximation of incoherence as an approximation on the accessible final states; this approxi- mation is believed to be particularly reliable for deepinelastic processes; it yields the convolution formula for the nuclear current tensor.

\section{A. Convolution formula for the nuclear current tensor}

Using the nuclear density operator $\rho_{A}\left(n_{A} P_{A}\right)$, the nuclear current tensor (3.1) takes the convolution form

$$
\left\langle n_{A}\left|W_{A}^{\mu \nu}\left(Q, P_{A}\right)\right| n_{A}\right\rangle=\sum_{t_{N}} \int d^{3} \mathbf{p}_{N} \frac{m_{N}}{p_{N}^{+}} \int_{-m_{A}+m_{A-1}}^{\infty} d M \operatorname{Tr}\left[W_{N\left(t_{N}\right)}^{\mu \nu}\left(Q_{N}(M), p_{N}\right) S\left(\mathbf{p}_{N} M t_{N}\right) \rho_{A}\left(n_{A} P_{A}\right)\right]
$$

in PWIA. Its spin matrix elements are

$$
\left\langle\lambda_{A}^{\prime}\left|W_{A}^{\mu \nu}\left(Q, P_{A}\right)\right| \lambda_{A}\right\rangle=\sum_{t_{N}} \int d^{3} \mathbf{p}_{N} \frac{m_{N}}{p_{N}^{+}} \int_{-m_{A}+m_{A-1}}^{\infty} d M \sum_{\lambda_{N} \lambda_{N}^{\prime}}\left\langle\lambda_{N}^{\prime}\left|W_{N\left(t_{N}\right)}^{\mu \nu}\left[Q_{N}(M), p_{N}\right]\right| \lambda_{N}\right\rangle\left\langle\lambda_{N} \lambda_{A}^{\prime}\left|S\left(\mathbf{p}_{N} M t_{N}\right)\right| \lambda_{N}^{\prime} \lambda_{A}\right\rangle .
$$

Because of the discussion on consistency at the beginning of this section, only the kinematic components $\mu, \nu=1,2,+$ will be taken from the convolution formula (5.2a). In Eq. (5.2a), $Q_{N}$ is the momentum transfer to the struck single nucleon; the latter momentum transfer

$$
Q_{N}(M):=\left\{Q^{-}+P_{A}^{-}-P_{A-1}^{-}\left(M+m_{A}\right)-p_{N}^{-}, \mathbf{Q}\right\}
$$

is different from the momentum transfer $Q$ to the whole nucleus. The momentum component $P_{A-1}^{-}\left(M+m_{A}\right)$ of the spectator $(A-1)$ nucleus is defined by $P_{A-1}^{-}\left(M+m_{A}\right)=\left[\left(M+m_{A}\right)^{2}+\mathbf{P}_{(A-1) \perp}^{2}\right] / P_{A-1}^{+}, M+m_{A}$ being the effective mass of the $(A-1)$ nucleus, $m_{A-1}$ is its minimum value. In Eq. (5.2) the nucleonic current tensor $W_{N\left(t_{N}\right)}^{\mu \nu}\left[Q_{N}(M), p_{N}\right]$ and the front form spectral function $S\left(\mathbf{p}_{N} M t_{N}\right)$ are introduced; their definitions will be discussed next. This section uses the capital $M$ in a notational meaning distinct from Sec. II.

\section{B. Nucleonic current tensor}

The nucleonic current tensor $W_{N\left(t_{N}\right)}^{\mu \nu}\left(Q_{N}, p_{N}\right)$ is defined by

$$
\begin{aligned}
\left\langle\lambda_{N}^{\prime}\left|W_{N\left(t_{N}\right)}^{\mu \nu}\left(Q_{N}, p_{N}\right)\right| \lambda_{N}\right\rangle= & (2 \pi)^{6} \frac{p_{N}^{+}}{m_{N}} \sum_{\beta_{1}} \int d^{3} \mathbf{p}_{x_{1}}\left\langle\mathbf{p}_{N} \lambda_{N}^{\prime} t_{N}\left|j_{N}^{\mu}(0)\right| \mathbf{p}_{x_{1}} \beta_{x_{1}}\right\rangle 2 \delta^{3}\left(\mathbf{p}_{x_{1}}-\mathbf{Q}-\mathbf{p}_{N}\right) \delta\left[p_{x_{1}}^{-}-Q_{N}^{-}(M)-p_{N}^{-}\right] \\
& \times\left\langle\mathbf{p}_{x_{1}} \beta_{x_{1}}\left|j_{N}^{\nu}(0)\right| \mathbf{p}_{N} \lambda_{N} t_{N}\right\rangle .
\end{aligned}
$$

It is an operator in nucleonic spin space and depends on isospin $t_{N}$. In Ref. [3] the corresponding instant form matrix elements are given. After transformation to front form spin states according to

$$
\left\langle\lambda_{N}^{\prime}\left|W_{N\left(t_{N}\right)}^{\mu \nu}\left(Q_{N}, p_{N}\right)\right| \lambda_{N}\right\rangle=\sum_{s_{N}^{\prime} s_{N}}\left\langle\lambda_{N}^{\prime}\left|R_{M}^{+}\left(p_{N}\right)\right| s_{N}^{\prime}\right\rangle\left\langle s_{N}^{\prime}\left|W_{N\left(t_{N}\right)}^{\mu \nu}\left(Q_{N}, p_{N}\right)\right| s_{N}\right\rangle\left\langle s_{N}\left|R_{M}\left(p_{N}\right)\right| \lambda_{N}\right\rangle,
$$

the nucleonic current tensor takes the form

$$
\begin{aligned}
\left\langle\lambda_{N}^{\prime}\left|W_{N\left(t_{N}\right)}^{\mu \nu}\left(Q_{N}, p_{N}\right)\right| \lambda_{N}\right\rangle= & \delta_{\lambda_{N^{\prime} \lambda_{N}}}\left[\left[\frac{Q_{N}^{\mu} Q_{N}^{\nu}}{Q_{N}^{2}}-g^{\mu \nu}\right] F_{1}^{N\left(t_{N}\right)}\left(x_{N}, Q_{N}^{2}\right) \frac{1}{m_{N}}+\frac{\widetilde{p}_{N}^{\mu} \widetilde{p}_{N}^{\nu}}{Q_{N} \cdot p_{N}} F_{2}^{N\left(t_{N}\right)}\left(x_{N}, Q_{N}^{2}\right) \frac{1}{m_{N}}\right] \\
& +i \frac{\epsilon^{\mu \nu \alpha \beta}}{Q_{N} \cdot p_{N}} Q_{N \alpha}\left\langle\lambda_{N}^{\prime}\left|\left[s_{\beta}\left(\vec{\sigma}_{N}\right) g_{1}^{N\left(t_{N}\right)}\left(x_{N}, Q_{N}^{2}\right)+\left(s_{\beta}\left(\vec{\sigma}_{N}\right)-\frac{Q_{N} \cdot s\left(\vec{\sigma}_{N}\right)}{Q_{N} \cdot p_{N}} p_{N \beta}\right) g_{2}^{N\left(t_{N}\right)}\left(x_{N}, Q_{N}^{2}\right)\right]\right| \lambda_{N}\right\rangle
\end{aligned}
$$

with the nucleonic Bjorken variable $x_{N}:=-Q_{N}^{2} / 2 Q_{N} \cdot p_{N}$ and with 


$$
\begin{gathered}
\widetilde{p}_{N}^{\mu}:=p_{N}^{\mu}-\frac{p_{N} \cdot Q_{N}}{Q_{N}^{2}} Q_{N}^{\mu}, \\
s\left(\vec{\sigma}_{N}\right):=\left\{\frac{m_{N}}{p_{N}^{+}}\left(\frac{\mathbf{p}_{N \perp}^{2}}{m_{N}^{2}} \hat{n} \cdot \vec{\sigma}_{N}+2 \frac{\mathbf{p}_{N \perp}}{m_{N}} \cdot \vec{\sigma}_{N \perp}-\hat{n} \cdot \vec{\sigma}_{N}\right), \vec{\sigma}_{N \perp}+\frac{\mathbf{p}_{N \perp}}{m_{N}} \hat{n} \cdot \vec{\sigma}_{N} \frac{p_{N}^{+}}{m_{N}} \hat{n} \cdot \vec{\sigma}_{N}\right\} .
\end{gathered}
$$

In fact, $s\left(\vec{\sigma}_{N}\right)$ acts like the Pauli-Lubanski vector $W$, except for factors, i.e., $\left\langle\lambda_{N}^{\prime}\left|s\left(\vec{\sigma}_{N}\right) / 2\right| \lambda_{N}\right\rangle=\left\langle\lambda_{N}^{\prime}\left|\left(1 / m_{N}\right) W\right| \lambda_{N}\right\rangle$.

\section{Spectral function}

The spectral function is defined as the probability of finding a nucleon of isospin $t_{N}$ with momentum $\mathbf{p}_{N}$ and the spectator $(A-1)$ nucleus with an excitation $f_{A-1}$ of mass $m_{A-1}\left(f_{A-1}\right)$, i.e.,

$$
\begin{aligned}
\left\langle\lambda_{N} \lambda_{A}^{\prime}\left|S\left(\mathbf{p}_{N} M t_{N}\right)\right| \lambda_{N}^{\prime} \lambda_{A}\right\rangle= & A \sum_{\lambda_{A-1} f_{A-1}} \delta\left[M+m_{A}-m_{A-1}\left(f_{A-1}\right)\right]\left\langle\mathbf{P}_{A} \lambda_{A}^{\prime} \mid \mathbf{p}_{N} \lambda_{N}^{\prime} t_{N}\left(\mathbf{P}_{A}-\mathbf{p}_{N}\right) \lambda_{A-1} f_{A-1}\right\rangle \\
& \times\left\langle\mathbf{p}_{N} \lambda_{N} t_{N}\left(\mathbf{P}_{A}-\mathbf{p}_{N}\right) \lambda_{A-1} f_{A-1} \mid \mathbf{P}_{A} \lambda_{A}\right\rangle .
\end{aligned}
$$

The spectral function is an operator in nucleonic and in nuclear spin space. Reference [18] defines it relativistically for the spin-averaged case; the extension to spin dependence is minor. The spectral function is calculated here in the nuclear c.m. system.

The spectral function is related to the front form momentum density $\rho\left(\mathbf{p}_{N} t_{N}\right)$ according to the sum rule

$$
\int d M\left\langle\lambda_{N} \lambda_{A}^{\prime}\left|S\left(\mathbf{p}_{N} M t_{N}\right)\right| \lambda_{N}^{\prime} \lambda_{A}\right\rangle=\left\langle\lambda_{N} \lambda_{A}^{\prime}\left|\rho\left(\mathbf{p}_{N} t_{N}\right)\right| \lambda_{N}^{\prime} \lambda_{A}\right\rangle
$$

with

$$
\left\langle\lambda_{N} \lambda_{A}^{\prime}\left|\rho\left(\mathbf{p}_{N} t_{N}\right)\right| \lambda_{N}^{\prime} \lambda_{A}\right\rangle=A \sum_{\lambda_{A-1} f_{A-1}}\left\langle\mathbf{P}_{A} \lambda_{A}^{\prime} \mid \mathbf{p}_{N} \lambda_{N}^{\prime} t_{N}\left(\mathbf{P}_{A}-\mathbf{p}_{N}\right) \lambda_{A-1} f_{A-1}\right\rangle\left\langle\mathbf{p}_{N} \lambda_{N} t_{N}\left(\mathbf{P}_{A}-\mathbf{p}_{N}\right) \lambda_{A-1} f_{A-1} \mid \mathbf{P}_{A} \lambda_{A}\right\rangle .
$$

The front form momentum density will be calculated in Sec. VI.

\section{Spin structure functions in deep-inelastic lepton scattering}

The results of the previous subsections are now adapted to deep-inelastic lepton scattering.

\section{Bjorken limit}

We consider the Bjorken limit $\left(Q^{0}\right)^{2} \gg-Q^{2} \gg m_{N}^{2}$ under the condition that the Bjorken variable $x=-A Q^{2} / 2 Q \cdot P_{A}$ remains constant. In terms of front form components for the momentum transfer, $\left(Q^{-}\right)^{2} \gg-Q^{2}$. In scattering from a many-nucleon system $Q_{N}^{-} \neq Q^{-}$, in principle; however, in the Bjorken $\operatorname{limit}_{\lim } Q_{N}=Q$ for all components [15]. In that limit the convolution formula for the nuclear current tensor of Eq. (5.2a) becomes

$$
\left\langle n_{A}\left|W_{A}^{\mu \nu}\left(Q, P_{A}\right)\right| n_{A}\right\rangle=\sum_{t_{N}} \int d^{3} \mathbf{p}_{N} \frac{m_{N}}{p_{N}^{+}} \operatorname{Tr}\left[W_{N\left(t_{N}\right)}^{\mu \nu}\left(Q, p_{N}\right) \rho\left(\mathbf{p}_{N} t_{N}\right) \rho_{A}\left(n_{A} P_{A}\right)\right] .
$$

The troublesome problem, that the nuclear current tensor $W_{A}^{\mu \nu}\left(Q, P_{A}\right)$ when calculated in PWIA does not respect current conservation, disappears in that limit, i.e., $Q_{\mu} W_{A}^{\mu \nu}\left(Q, P_{A}\right)=W_{A}^{\mu \nu}\left(Q, P_{A}\right) Q_{\nu}=0$. In the Bjorken limit the spin-structure functions scale; experimentally one should see them to become independent from $Q^{2}$. The convolution formula (5.9) yields that scaling behavior; the nucleonic structure functions only depend on the nucleonic Bjorken variable $x_{N}$ and the resulting nuclear structure functions will also turn out to depend on the nuclear Bjorken variable $x$ only; the dependence of the spin-structure functions $g_{i}^{N\left(t_{N}\right)}$ and $g_{i}^{A}$ on the momentum transfers $Q_{N}^{2}$ and $Q^{2}$, respectively, will therefore be scratched from now on. The two Bjorken variables are related by

$$
x_{N}=x \frac{1}{A} \frac{Q \cdot P_{A}}{Q \cdot p_{N}}=x \frac{P_{A}^{+}}{A p_{N}^{+}},
$$

the momentum fraction $p_{N}^{+} / P_{A}^{+}=x / A x_{N}$ will often be needed. 


\section{Extraction from selected current tensor matrix elements}

Extraction scheme I of Sec. III D only needs kinematic components of the nuclear current tensor; it can therefore be employed consistently in PWIA; it is our favorite extraction scheme in this paper. Using the convolution formula (5.9) for the matrix element (3.14) of the nuclear current tensor, i.e.,

$$
\begin{aligned}
e_{1 \mu} e_{2 \nu}\left\langle n_{A}\left|W_{A}^{\mu \nu}\left(Q, P_{A}\right)\right| n_{A}\right\rangle= & i A \sum_{t_{N}} \int d^{3} \mathbf{p}_{N} \frac{m_{N}}{p_{N}^{+}} \frac{1}{p_{N}^{+}} \operatorname{Tr}\left[\left(n \cdot s\left(\vec{\sigma}_{N}\right) g_{1}^{N\left(t_{N}\right)}\left(x_{N}\right)-2 \frac{\sqrt{-Q^{2}}}{Q^{-}} e_{1} \cdot s\left(\vec{\sigma}_{N}\right) g_{2}^{N\left(t_{N}\right)}\left(x_{N}\right)\right)\right. \\
& \left.\times \rho\left(\mathbf{p}_{N} t_{N}\right) \rho_{A}\left(n_{A} P_{A}\right)\right],
\end{aligned}
$$

convolution formulas result for $g_{i}^{A}$ in the form

$$
\begin{gathered}
g_{1}^{A}(x)=\left.\sum_{t_{N}} \int \frac{d \xi}{A \xi} g_{1}^{N\left(t_{N}\right)}\left(\frac{x}{A \xi}\right) \vec{n}_{A} \cdot \vec{s}_{N}\left(\xi t_{N} n_{A}\right)\right|_{n_{A}} \| \hat{n} \\
g_{2}^{A}(x)=\left.\sum_{t_{N}} \int \frac{d \xi}{A \xi} \frac{1}{A \xi} g_{2}^{N\left(t_{N}\right)}\left(\frac{x}{A \xi}\right) \vec{n}_{A} \cdot \vec{s}_{N}\left(\xi t_{N} n_{A}\right)\right|_{n_{A}} \| \hat{e}_{1}
\end{gathered}
$$

provided the front form spin distribution is defined by

$\vec{s}_{N}\left(\xi t_{N} n_{A}\right):=\int d^{3} \mathbf{p}_{N} \delta\left(\xi-\frac{p_{N}^{+}}{P_{A}^{+}}\right) \operatorname{Tr}\left[\vec{\sigma}_{N} \rho\left(\mathbf{p}_{N} t_{N}\right) \rho_{A}\left(n_{A} P_{A}\right)\right]$.

The assumption $\vec{n}_{A} \| \hat{n}$ is essential for the derivation of $g_{1}^{A}$, the assumption $\vec{n}_{A} \| \hat{e}_{1}$ is essential for the derivation of $g_{2}^{A}$. The front form spin distribution $\vec{s}_{N}\left(\xi t_{N} n_{A}\right)$ will be calculated in Sec. VI for the three-nucleon bound state and in Appendix $\mathrm{C}$ for the deuteron. It takes on the role which the spinaveraged front form momentum distribution $f_{N}\left(\xi t_{N}\right)$, i.e.,

$$
f_{N}\left(\xi t_{N}\right):=\int d^{3} \mathbf{p}_{N} \delta\left(\xi-\frac{p_{N}^{+}}{P_{A}^{+}}\right) \operatorname{Tr}\left[\rho\left(\mathbf{p}_{N} t_{N}\right) \rho_{A}\left(n_{A} P_{A}\right)\right],
$$

played for the calculation of the spin-independent structure function $F_{2}^{A}$ in Ref. [18]. Normalization and momentum conservation implied

$$
\begin{aligned}
& \sum_{t_{N}} \int d \xi f_{N}\left(\xi t_{N}\right)=A \\
& \sum_{t_{N}} \int d \xi \xi f_{N}\left(\xi t_{N}\right)=1 .
\end{aligned}
$$

The front form spin distribution $\vec{s}_{N}\left(\xi t_{N} n_{A}\right)$ also satisfies a sum rule, i.e.,

$$
\int d \xi \vec{n}_{A} \cdot \vec{s}_{N}\left(\xi t_{N} n_{A}\right)=\left\langle\sigma_{N\left(t_{N}\right)}\right\rangle
$$

Thus, it is related to the spin expectation value $\left\langle\sigma_{N\left(t_{N}\right)}\right\rangle$ in the polarized trinucleon bound state $\left|\mathbf{P}_{A} n_{A}\right\rangle=\left|\Psi_{B} n_{A}\right\rangle$, defined by

$$
\left\langle\sigma_{N\left(t_{N}\right)}\right\rangle:=\left\langle\Psi_{B} n_{A}\left|\sum_{i=1}^{3}\left(\frac{1}{2}+t_{N} \tau_{N}(i)\right) \vec{n}_{A} \cdot \vec{\sigma}_{N}(i)\right| \Psi_{B} n_{A}\right\rangle,
$$

i.e., to the nucleonic contribution to the nuclear spin, $\vec{\sigma}_{N}(i)$ and $\tau_{N}(i)$ being the nucleonic spin and isospin projection operators. The spin expectation value $\left\langle\sigma_{N\left(t_{N}\right)}\right\rangle$ is given by the $S$-, $S^{\prime}-$, and $D$-state probabilities $P(S), P\left(S^{\prime}\right)$, and $P(D)$ of the trinucleon wave function according to

$$
\left\langle\sigma_{N\left(t_{N}\right)}\right\rangle= \begin{cases}\frac{2}{3}\left[P\left(S^{\prime}\right)-P(D)\right], & t_{N}=+\frac{1}{2} \text { (proton), } \\ P(S)+\frac{1}{3}\left[P\left(S^{\prime}\right)-P(D)\right], & t_{N}=-\frac{1}{2} \text { (neutron). }\end{cases}
$$

The sum rule is calculated in the nuclear c.m. system for which $n_{A}=\left(0, \hat{n}_{R}\right)$; it does not depend on the direction of the polarization $n_{A}$.

\section{Extraction from the full current tensor}

Extraction scheme II of Sec. III D requires the full nuclear current tensor which cannot be calculated consistently in PWIA. We only discuss the extraction according to Eqs. (3.16a) and (3.16b) for curiosity, since it was used in Ref. [3] for nonrela- 
tivistic phenomena. The extraction scheme II yields

$$
\begin{aligned}
g_{1}^{A}(x)= & -\frac{1}{A} \sum_{t_{N}} \int d^{3} \mathbf{p}_{N} \frac{m_{N}}{p_{N}^{+}} \frac{Q \cdot P_{A}}{P_{A}^{2}\left[Q^{2}+\left(Q \cdot n_{A}\right)^{2}\right]-\left(Q \cdot P_{A}\right)^{2}} \frac{P_{A}}{Q \cdot p_{N}} \operatorname{Tr}\left(\left\{n_{A} \cdot s\left(\vec{\sigma}_{N}\right)-\frac{Q \cdot P_{A}}{P_{A}^{2}} \frac{P_{A} \cdot s\left(\vec{\sigma}_{N}\right)}{Q \cdot n_{A}}\right.\right. \\
& \left.\left.\left.-\left(\frac{\left(Q \cdot n_{A}\right)^{2}}{Q^{2}}-\frac{\left(Q \cdot P_{A}\right)^{2}}{P_{A}^{2} Q^{2}}\right) \frac{Q \cdot s\left(\vec{\sigma}_{N}\right)}{Q \cdot n_{A}}\right] g_{1}^{N\left(t_{N}\right)}\left(x_{N}\right)+\left[n_{A} \cdot \widetilde{s}\left(\vec{\sigma}_{N}\right)-\frac{Q \cdot P_{A}}{P_{A}^{2}} \frac{P_{A} \cdot \widetilde{s}\left(\vec{\sigma}_{N}\right)}{Q \cdot n_{A}}\right] g_{2}^{N\left(t_{N}\right)}\left(x_{N}\right)\right\} \rho\left(\mathbf{p}_{N} t_{N}\right) \rho_{A}\left(n_{A} P_{A}\right)\right), \\
g_{2}^{A}(x)= & \frac{1}{A} \sum_{t_{N}} \int d^{3} \mathbf{p}_{N} \frac{m_{N}}{p_{N}^{+}} \frac{\left(Q \cdot P_{A}\right)^{2}}{P_{A}^{2}\left[Q^{2}+\left(Q \cdot n_{A}\right)^{2}\right]-\left(Q \cdot P_{A}\right)^{2}} \frac{Q \cdot P_{A}}{Q \cdot p_{N}} \operatorname{Tr}\left(\left\{\left[n_{A} \cdot s\left(\vec{\sigma}_{N}\right)-\frac{Q^{2}+\left(Q \cdot n_{A}\right)^{2}}{Q \cdot P_{A}} \frac{P_{A} \cdot s\left(\vec{\sigma}_{N}\right)}{Q \cdot n_{A}}+\frac{Q \cdot s\left(\vec{\sigma}_{N}\right)}{Q \cdot n_{A}}\right]\right.\right. \\
& \left.\left.\times g_{1}^{N\left(t_{N}\right)}\left(x_{N}\right)+\left[n_{A} \cdot \widetilde{s}\left(\vec{\sigma}_{N}\right)-\frac{Q^{2}+\left(Q \cdot n_{A}\right)^{2}}{Q \cdot P_{A}} \frac{P_{A} \cdot \widetilde{s}\left(\vec{\sigma}_{N}\right)}{Q \cdot n_{A}}\right] g_{2}^{N\left(t_{N}\right)}\left(x_{N}\right)\right\} \rho\left(\mathbf{p}_{N} t_{N}\right) \rho_{A}\left(n_{A} P_{A}\right)\right) .
\end{aligned}
$$

The abbreviation

$$
\widetilde{s}\left(\vec{\sigma}_{N}\right):=s\left(\vec{\sigma}_{N}\right)-\frac{Q \cdot s\left(\vec{\sigma}_{N}\right)}{Q \cdot p_{N}} p_{N}
$$

is introduced in Eqs. (5.17a) and (5.17b). The choice of polarization for $n_{A}$ and the approach of the Bjorken limit are noncommuting mathematical procedures. It seems to make sense to take the Bjorken limit only after a definite polarization $n_{A}$ is assumed. In case of the polarization $\vec{n}_{A} \| \hat{n}$, Eq. (5.17a) yields the convolution formula Eq. (5.12a) for the spin-structure function $g_{1}^{A}$ in the Bjorken limit; in case of the polarization $\vec{n}_{A} \| \hat{e}_{1}$, Eq. (5.17b) yields the convolution formula Eq. (5.12b) for the spin-structure function $g_{2}^{A}$ in the Bjorken limit. Thus, there appears to be complete consistency between the two employed extraction schemes. We shall therefore use the convolution formulas (5.12a) and (5.12b) in all applications of this paper. However, that consistency is not general.

Under the assumption $\vec{n}_{A} \| \hat{e}_{1}$ an alternative convolution formula for $g_{1}^{A}$ results from Eq. (5.17a) in the Bjorken limit; under the assumption $\vec{n}_{A} \| \hat{n}$ an alternative convolution formula for $g_{2}^{A}$ results from Eq. (5.17b) in the Bjorken limit, i.e.,

$$
\begin{aligned}
g_{1}^{A}(x) & =\frac{1}{A} \sum_{t_{N}} \int d^{3} \mathbf{p}_{N} \frac{m_{N}}{p_{N}^{+}} \frac{P_{A}^{+}}{p_{N}^{+}} \operatorname{Tr}\left[\left\{\hat{e}_{1} \cdot \vec{\sigma}_{N}+\hat{n} \cdot \vec{\sigma}_{N} \frac{\hat{e}_{1} \cdot \mathbf{p}_{N \perp}}{m_{N}}\right\} g_{1}^{N\left(t_{N}\right)}\left(x_{N}\right) \rho\left(\mathbf{p}_{N} t_{N}\right) \rho_{A}\left(n_{A} P_{A}\right)\right], \\
g_{2}^{A}(x)= & \frac{1}{A} \sum_{t_{N}} \int d^{3} \mathbf{p}_{N}\left(\frac{m_{N}}{p_{N}^{+}}\right)^{2} \frac{P_{A}^{+}}{p_{N}^{+}} \operatorname{Tr}\left[\left\{\hat{n} \cdot \vec{\sigma}_{N}\left[1-\left(\frac{p_{N}^{+}}{m_{N}}\right)^{2}+\frac{\mathbf{p}_{N \perp}^{2}}{p_{N}^{+} m_{N}}\right]+\frac{p_{N}^{+} \mathbf{p}_{N \perp} \cdot \vec{\sigma}_{N}}{m_{N}^{2}}\right\} g_{1}^{N\left(t_{N}\right)}\left(x_{N}\right)\right. \\
& \left.\left.+\left[\hat{n} \cdot \vec{\sigma}_{N}\left(1+\frac{\left(\hat{e}_{1} \cdot \mathbf{p}_{N}\right)^{2}}{m_{N}^{2}}\right)-\frac{\mathbf{p}_{N \perp} \cdot \vec{\sigma}_{N}}{p_{N}^{+}}\right] g_{2}^{N\left(t_{N}\right)}\left(x_{N}\right)\right) \rho\left(\mathbf{p}_{N} t_{N}\right) \rho_{A}\left(n_{A} P_{A}\right)\right] .
\end{aligned}
$$

The alternative convolution formulas Eqs. (5.19a) and (5.19b), are structurely different from those of Eqs. (5.12a) and (5.12b). They contain terms proportional to $\mathbf{p}_{N \perp}$ which cannot be expressed by the front form spin distribution $\vec{s}_{N}\left(\xi t_{N} n_{A}\right)$. Though the spin-structure functions $g_{1}^{A}$ and $g_{2}^{A}$ are Lorentz scalars, independent from the nuclear polarization, the ones approximately calculated remain dependent. We notice that dependence with amazement, especially, since in the instant form description of quasielastic scattering no such dependence arises. However, we have checked the quantitative differences of the convolution formulas (5.12a) and (5.19a) for $g_{1}^{A}$; the differences are not resolvable on the plots shown later.

\section{FRONT FORM MOMENTUM DENSITY OF THE TRINUCLEON BOUND STATE AND SPIN DISTRIBUTION}

In this section the front form momentum density $\rho\left(\mathbf{p}_{N} t_{N}\right)$ of Eq. (5.8b) is calculated for the trinucleon bound state, i.e., for $A=3$. It is calculated as an operator in nucleonic and nuclear spin space. Introducing the spin-dependent operator

$$
\left\langle\lambda_{i}^{\prime}\left|O_{i}\left(\lambda_{N}^{\prime} \lambda_{N}\right)\right| \lambda_{i}\right\rangle:=\delta_{\lambda_{i}^{\prime} \lambda_{N}^{\prime}} \delta_{\lambda_{i} \lambda_{N}}
$$

for nucleon $i$ by its matrix elements, the front form momentum density $\rho\left(\mathbf{p}_{N} t_{N}\right)$ is related to the nuclear bound state $\left|\mathbf{P}_{A} \lambda_{A}\right\rangle$ with spin projection $\lambda_{A}$, i.e.,

$$
\left\langle\lambda_{N} \lambda_{A}^{\prime}\left|\rho\left(\mathbf{p}_{N} t_{N}\right)\right| \lambda_{N}^{\prime} \lambda_{A}\right\rangle=\left\langle\mathbf{P}_{A} \lambda_{A}^{\prime}\left|\sum_{i} \delta^{3}\left(\mathbf{p}_{N}-\mathbf{p}_{i}\right) O_{i}\left(\lambda_{N}^{\prime} \lambda_{N}\right) \delta_{t_{N} t_{i}}\right| \mathbf{P}_{A} \lambda_{A}\right\rangle,
$$




$$
\left\langle\lambda_{N} \lambda_{A}^{\prime}\left|\rho\left(\mathbf{p}_{N} t_{N}\right)\right| \lambda_{N}^{\prime} \lambda_{A}\right\rangle=\frac{A}{P_{A}^{+}}\left\langle\mathbf{P}_{A} \lambda_{A}^{\prime}\left|\delta\left(\frac{p_{N}^{+}}{P_{A}^{+}}-\frac{p_{1}^{+}}{P_{A}^{+}}\right) \delta^{2}\left(\mathbf{p}_{N \perp}-\mathbf{p}_{1 \perp}\right) O_{1}\left(\lambda_{N}^{\prime} \lambda_{N}\right) \delta_{t_{N} t_{1}}\right| \mathbf{P}_{A} \lambda_{A}\right\rangle .
$$

In the operator the front form momentum fraction $p_{N}^{+} / P_{A}^{+}$is introduced.

The single-nucleon front form momenta $\mathbf{p}_{i}$ are related to the c.m. momentum $\mathbf{P}_{A}$ and to the internal front form momenta $\mathbf{k}_{i \perp}$ and the front form momentum fractions $\xi_{i}$ by

$$
\begin{gathered}
\mathbf{P}_{A \perp}=\sum_{j} \mathbf{p}_{j \perp}, \\
P_{A}^{+}=\sum_{j} p_{j}^{+}, \\
\mathbf{k}_{i \perp}=\mathbf{p}_{i \perp}-\frac{p_{i}^{+}}{P_{A}^{+}} \mathbf{P}_{A \perp}, \\
\xi_{i}=\frac{p_{i}^{+}}{P_{A}^{+}} .
\end{gathered}
$$

The internal single-nucleon momenta $\left(\mathbf{k}_{i \perp} \xi_{i}\right)$ satisfy the two constraints $\Sigma_{i} \mathbf{k}_{i \perp}=0$ and $\Sigma_{i} \xi_{i}=1$. For the calculation of the front form momentum density the nuclear c.m. system is assumed, i.e., $\mathbf{P}_{A \perp}=0$ and $P_{A}^{+}=m_{A}$. The internal part of the nuclear bound state $\left|\mathbf{P}_{A} \lambda_{A}\right\rangle$ is a mass eigenstate as in Eq. (2.11), a proper state of relativistic quantum mechanics; however, when the interactions are added to the square of the free mass operator, the resulting eigenvalue equation becomes [16] formally identical with the eigenvalue equation for an internal nonrelativistic Hamiltonian. Thus, the relativistic mass eigenstate can be identified with the nonrelativistic ground state $\left|\Psi_{B} \lambda_{A}\right\rangle$ of internal motion; it was already used in Eq. (5.16a). The mass eigenstate is independent of any choice of the dynamics; it is represented here in terms of front form variables for momenta and spin. However, that identification of $\left|P_{A} \lambda_{A}\right\rangle$ with $\left|\Psi_{B} \lambda_{A}\right\rangle$ has one serious drawback: Rotational invariance is violated, since $\left|\Psi_{B} \lambda_{A}\right\rangle$ is an eigenstate of an angular momentum operator, built up from the operators of constituents without interactions. That violation [17] gives rise to the well-known ambiguities when elastic form factors are extracted from approximately computed current matrix elements. The same ambiguities are likely to reoccur in the present context when extracting structure functions from an approximately computed current tensor. This paper does not explore those ambiguities in full, but at least it compares two extraction schemes according to Secs. III D, V D 2, and V D 3 which weight spin matrix elements differently, but which yield spin-structure functions numerically almost indistinguishable. In view of that shortcoming, the suggestion of Ref. [19] to construct relativistic mass eigenstates directly is an interesting alternative, but it has not yet matured such that it can be employed simultaneously in the two- and three-nucleon systems.
The internal single-nucleon momenta $\left(\mathbf{k}_{i \perp} \xi_{i}\right)$ are extended to the standard three-dimensional form of on mass shell particles

$$
\begin{gathered}
k_{i}^{+}=\xi_{i} M_{0}, \\
k_{i}^{-}=\frac{m_{i}^{2}+\mathbf{k}_{i \perp}^{2}}{k_{i}^{+}}, \\
\vec{k}_{i}=\left[k_{i}^{1}, k_{i}^{2}, \frac{1}{2}\left(\xi_{i} M_{0}-\frac{m_{i}^{2}+\mathbf{k}_{i \perp}^{2}}{\xi_{i} M_{0}}\right)\right]
\end{gathered}
$$

with

$$
M_{0}=\left(\sum_{j} \frac{m_{j}^{2}+\mathbf{k}_{j \perp}^{2}}{\xi_{j}}\right)^{1 / 2}
$$

and $k_{i}^{2}=m_{i}^{2}$. That three-dimensional form $\vec{k}_{i}$ is also constrained by $\Sigma_{i} \vec{k}_{i}=0$. The internal momenta $\vec{k}_{i}$ are therefore related to the Jacobi momenta $(\vec{p} \vec{q})$, in which the ground state $\left|\Psi_{B} \lambda_{A}\right\rangle$ usually is given, by

$$
\begin{gathered}
\vec{p}=\frac{1}{2}\left(\vec{k}_{2}-\vec{k}_{3}\right), \\
\vec{q}=-\vec{k}_{1},
\end{gathered}
$$

assuming equal masses $m_{i}=m_{N}$ for the three nucleons. Thus, a complete transformation of the wave function to the internal front form momenta $\left(\mathbf{k}_{i \perp} \xi_{i}\right)$ and the front form spins is possible. However, the front form momentum density $\rho\left(\mathbf{p}_{N} t_{N}\right)$ samples the front form properties of a single nucleon according to Eq. (6.2b). Thus, a mixed set of momenta $\left(\vec{p} \mathbf{k}_{1 \perp} \xi_{1}\right)$ is preferable in terms of which the Jacobi momenta can be expressed, i.e.,

$$
\begin{gathered}
\vec{p}=\vec{p}, \\
\vec{q}=\left[-k_{1}^{1},-k_{1}^{2},-\frac{1}{2}\left(\xi_{1} M_{0}-\frac{m_{N}^{2}+\mathbf{k}_{1 \perp}^{2}}{\xi_{1} M_{0}}\right)\right] .
\end{gathered}
$$

In this representation the multicoordinate quantity $M_{0}$ becomes

$$
M_{0}=\left[\frac{4\left(m_{N}^{2}+p^{2}\right)+\mathbf{k}_{1 \perp}^{2}}{1-\xi_{1}}+\frac{m_{N}^{2}+\mathbf{k}_{1 \perp}^{2}}{\xi_{1}}\right]^{1 / 2}
$$

with the notation $p^{2}=\vec{p}^{2}$ in the remainder of this section. Equation (6.6c) holds only approximately; it neglects angledependent terms $\vec{p} \cdot \mathbf{k}_{1 \perp} / m_{N}^{2}$ which are, however, small for the physically relevant momenta in a nuclear bound state. The relativistic trinucleon bound state wave function takes the following form in that mixed representation: 


$$
\left\langle\vec{p} \mathbf{k}_{1 \perp} \xi_{1} \lambda_{1} s_{2} s_{3} t_{1} t_{2} t_{3} \mid \Psi_{B} \lambda_{A}\right\rangle=\sum_{s_{1}}\left\langle\lambda_{1}\left|R_{M}^{+}\left(\mathbf{k}_{1 \perp} \xi_{1} p^{2}\right)\right| s_{1}\right\rangle \sqrt{\left|\frac{\partial(\vec{p} \vec{q})}{\partial\left(\vec{p} \mathbf{k}_{1 \perp} \xi_{1}\right)}\right|}\left\langle\vec{p} \vec{q}\left(\mathbf{k}_{1 \perp} \xi_{1} p^{2}\right) s_{1} s_{2} s_{3} t_{1} t_{2} t_{3} \mid \Psi_{B} \lambda_{A}\right\rangle .
$$

The transformation of momenta requires the Jacobi determinant

$$
\left|\frac{\partial(\vec{p} \vec{q})}{\partial\left(\vec{p} \mathbf{k}_{1 \perp} \xi_{1}\right)}\right|=\left|\frac{\partial q^{3}}{\partial \xi_{1}}\left(\mathbf{k}_{1 \perp} \xi_{1} p^{2}\right)\right|=\frac{M_{0}\left(\mathbf{k}_{1 \perp} \xi_{1} p^{2}\right)}{4 \xi_{1}\left(1-\xi_{1}\right)}
$$

in Eq. (6.8) the argument list of $M_{0}$ is made explicit. Only the spin of nucleon 1 is Melosh rotated. The rotation matrix $R_{M}$ depends on $k_{1}$, but through $M_{0}$ on all internal variables $\left(\mathbf{k}_{i \perp} \xi_{i}\right)$, therefore also on $p^{2}$; this is the reason why its argument $k_{1}$ is changed to the argument list $\left(\mathbf{k}_{1 \perp} \xi_{1} p^{2}\right)$, i.e.,

$$
R_{M}\left(\mathbf{k}_{1 \perp} \xi_{1} p^{2}\right)=\frac{m_{N}+\xi_{1} M_{0}+i\left(\sigma_{N}^{1} k_{1}^{2}-\sigma_{N}^{2} k_{1}^{1}\right)}{\sqrt{\left(m_{N}+\xi_{1} M_{0}\right)^{2}+\mathbf{k}_{1 \perp}^{2}}},
$$

in contrast to the notation in Eq. (2.15c). The spins of the nucleons 2 and 3 are still taken to be canonical, since the expectation value $(6.2 b)$ sums over that spin dependence.

The front form momentum density is calculated according to

$$
\begin{aligned}
\left\langle\lambda_{N} \lambda_{A}^{\prime}\left|\rho\left(\mathbf{p}_{N} t_{N}\right)\right| \lambda_{N}^{\prime} \lambda_{A}\right\rangle= & \frac{A}{P_{A}^{+}} \sum_{t_{2} t_{3}} \sum_{s_{1}^{\prime} s_{1} s_{2} s_{3}} \int d^{3} \vec{p}\left|\frac{\partial q^{3}}{\partial \xi}\left(\mathbf{p}_{N \perp} \xi p^{2}\right)\right|\left\langle\lambda_{N}\left|R_{M}^{+}\left(\mathbf{p}_{N \perp} \xi p^{2}\right)\right| s_{1}\right\rangle\left\langle s_{1}^{\prime}\left|R_{M}\left(\mathbf{p}_{N \perp} \xi p^{2}\right)\right| \lambda_{N}^{\prime}\right\rangle \\
& \times\left.\left\langle\Psi_{B} \lambda_{A}^{\prime} \mid \vec{p} \vec{q}\left(\mathbf{p}_{N \perp} \xi p^{2}\right) s_{1}^{\prime} s_{2} s_{3} t_{N} t_{2} t_{3}\right\rangle\left\langle\vec{p} \vec{q}\left(\mathbf{p}_{N \perp} \xi p^{2}\right) s_{1} s_{2} s_{3} t_{N} t_{2} t_{3} \mid \Psi_{B} \lambda_{A}\right\rangle\right|_{\xi=p_{N}^{+} / P_{A}^{+}}
\end{aligned}
$$

At this stage we go back to the corresponding instant form momentum density $\rho\left(\vec{p}_{N} t_{N}\right)$ which reads, in the nuclear c.m. system,

$$
\begin{aligned}
\left\langle s_{N} s_{A}^{\prime}\left|\rho\left(\vec{p}_{N} t_{N}\right)\right| s_{N}^{\prime} s_{A}\right\rangle & =\left\langle\Psi_{B} s_{A}^{\prime}\left|\sum_{i} \delta\left(\vec{p}_{N}-\vec{k}_{i}\right) O_{i}\left(s_{N}^{\prime} s_{N}\right) \delta_{t_{N} t_{i}}\right| \Psi_{B} s_{A}\right\rangle \\
& =A \sum_{t_{2} t_{3}} \sum_{s_{2} s_{3}} \int d^{3} \vec{p}\left\langle\Psi_{B} s_{A}^{\prime} \mid \vec{p}\left(-\vec{p}_{N}\right) s_{N}^{\prime} s_{2} s_{3} t_{N} t_{2} t_{3}\right\rangle\left\langle\vec{p}\left(-\vec{p}_{N}\right) s_{N} s_{2} s_{3} t_{N} t_{2} t_{3} \mid \Psi_{B} s_{A}\right\rangle \\
& =\frac{1}{2}\left\langle s_{N} s_{A}^{\prime}\left|\rho_{0}\left(p_{N} t_{N}\right)+\rho_{1}\left(p_{N} t_{N}\right) \vec{\sigma}_{N} \cdot \vec{\sigma}_{A}+\rho_{2}\left(p_{N} t_{N}\right)\left(\frac{\vec{\sigma}_{N} \cdot \vec{p}_{N} \vec{\sigma}_{A} \cdot \vec{p}_{N}}{\vec{p}_{N}^{2}}-\frac{1}{3} \vec{\sigma}_{N} \cdot \vec{\sigma}_{A}\right)\right| s_{N}^{\prime} s_{A}\right\rangle \\
& =\frac{1}{2} \int p^{2} d p\left\langle s_{N} s_{A}^{\prime}\left|\bar{\rho}_{0}\left(p_{N} p t_{N}\right)+\bar{\rho}_{1}\left(p_{N} p t_{N}\right) \vec{\sigma}_{N} \cdot \vec{\sigma}_{A}+\bar{\rho}_{2}\left(p_{N} p t_{N}\right)\left(\frac{\vec{\sigma}_{N} \cdot \vec{p}_{N} \vec{\sigma}_{A} \cdot \vec{p}_{N}}{\vec{p}_{N}^{2}}-\frac{1}{3} \vec{\sigma}_{N} \cdot \vec{\sigma}_{A}\right)\right| s_{N}^{\prime} s_{A}\right\rangle .
\end{aligned}
$$

The first step gives the definition. In the next two steps of Eq. (6.11) the spin structure of the instant form momentum density $\rho\left(\vec{p}_{N} t_{N}\right)$ is recalled; in the last step it is realized that that spin structure already holds after integration on $\hat{p}$, i.e., prior to the integration on the magnitude $p$ of $\vec{p}$. The functions $\bar{\rho}_{0}, \bar{\rho}_{1}$, and $\bar{\rho}_{2}$ in the result $(6.11)$ are numerically available and will be used for calculating the front form momentum density. Since the calculation is carried out in the nuclear c.m. system, the canonical and front form spins of the nucleus are identical, thus, $\left|\Psi_{B} \lambda_{A}\right\rangle=\left|\Psi_{B} s_{A}\right\rangle$ and therefore

$$
\begin{aligned}
\left\langle\lambda_{N} \lambda_{A}^{\prime}\left|\rho\left(\mathbf{p}_{N} t_{N}\right)\right| \lambda_{N}^{\prime} \lambda_{A}\right\rangle= & \frac{1}{2} \frac{1}{P_{A}^{+}} \int p^{2} d p\left|\frac{\partial q^{3}}{\partial \xi}\left(\mathbf{p}_{N \perp} \xi p^{2}\right)\right|\left\langle\lambda_{N} \lambda_{A}^{\prime}\left|R_{M}^{+}\left(\mathbf{p}_{N \perp} \xi p^{2}\right)\right| \bar{\rho}_{0}\left(q p t_{N}\right)+\bar{\rho}_{1}\left(q p t_{N}\right) \vec{\sigma}_{N} \cdot \vec{\sigma}_{A}+\bar{\rho}_{2}\left(q p t_{N}\right)\right. \\
& \left.\times\left(\frac{\vec{\sigma}_{N} \cdot \vec{q} \vec{\sigma}_{A} \cdot \vec{q}}{\vec{q}^{2}}-\frac{1}{3} \vec{\sigma}_{N} \cdot \vec{\sigma}_{A}\right)\right]\left.\left.\right|_{\vec{q}=\vec{q}\left(\mathbf{p}_{N \perp} \xi p^{2}\right)} R_{M}\left(\mathbf{p}_{N \perp} \xi p^{2}\right)\left|\lambda_{N}^{\prime} \lambda_{A}\right|\right|_{\xi=p_{N}^{+} / P_{A}^{+}} .
\end{aligned}
$$

The result (6.12) requires the replacement of the Jacobi momentum $\vec{q}$ as $\vec{q}\left(\mathbf{p}_{N \perp} \xi p^{2}\right)$ by the front form momenta ( $\mathbf{p}_{N \perp} \xi$ ) according to the prescription of Eq. $(6.6 \mathrm{~b})$, given there in terms of $\left(\mathbf{k}_{1 \perp} \xi_{1}\right)$. 
The determination of the spin-structure functions $g_{1}^{A}$ and $g_{2}^{A}$ in deep-inelastic lepton scattering according to Eqs. (5.19) requires the full spin-dependent momentum density $\rho\left(\mathbf{p}_{N} t_{N}\right)$; however, the determination according to Eqs. (5.12) which this paper prefers needs the front form spin distribution $\vec{s}_{N}\left(\xi t_{N} n_{A}\right)$ only, i.e.,

$$
\begin{aligned}
\vec{s}_{N}\left(\xi t_{N} n_{A}\right)= & \frac{1}{2} \int d^{2} \mathbf{p}_{N \perp} \int p^{2} d p\left|\frac{\partial q^{3}}{\partial \xi}\left(\mathbf{p}_{N \perp} \xi p^{2}\right)\right| \sum_{i=1}^{3} \operatorname{Tr}\left[\vec{\sigma}_{N} R_{M}^{+}\left(\mathbf{p}_{N \perp} \xi p^{2}\right) \sigma_{N}^{i} R_{M}\left(\mathbf{p}_{N \perp} \xi p^{2}\right)\right] \\
& \times\left.\left[\bar{\rho}_{1}\left(q p t_{N}\right) n_{A}^{i}+\bar{\rho}_{2}\left(p_{N} p t_{N}\right)\left(\frac{q^{i} \vec{n}_{A} \cdot \vec{q}}{\vec{q}^{2}}-\frac{1}{3} n_{A}^{i}\right)\right]\right|_{\vec{q}=\vec{q}\left(\mathbf{p}_{N \perp} \xi p^{2}\right)} .
\end{aligned}
$$

The front form spin distribution $\vec{s}_{N}\left(\xi t_{N} n_{A}\right)$ was already introduced in Eq. (5.13). The calculations of this paper are based on it.

\section{RESULTS AND DISCUSSION}

The spin-structure functions $g_{1}^{A}$ and $g_{2}^{A}$ of deep-inelastic lepton scattering are calculated according to Eqs. (5.12). We use the theoretical apparatus developed for the deuteron in Appendices A-C as well and therefore discuss deep-inelastic lepton scattering from the deuteron and the three-nucleon bound states simultaneously. We shall abbreviate the label $N\left(t_{N}\right)$ from now on by $p$ and $n$ for $t_{N}=+\frac{1}{2}$ and $t_{N}=-\frac{1}{2}$, differentiating proton and neutron contributions, respectively.

\section{A. Front form spin distribution}

The deuteron and ${ }^{3} \mathrm{He}$ front form spin distributions $\vec{s}_{N}\left(\xi t_{N} n_{A}\right)$ are displayed as functions of the momentum fraction $\xi$ in Figs. 1 and 2. The components $\vec{n}_{A} \cdot \vec{s}_{N}\left(\xi t_{N} n_{A}\right)$ are given for longitudinal and transverse polarizations, i.e., for $\vec{n}_{A}=\hat{n}$ and $\vec{n}_{A}=\hat{e}_{1}$, respectively. They are split into proton and neutron contributions. The calculation is based on relativistic mass eigenstates which are obtained [16] by reinterpreting nonrelativistic bound states. Their wave functions are derived from the Paris potential [20] for the deuteron and for the trinucleon bound state.

\section{Deuteron}

The employed relativitic deuteron wave function is obtained from a nonrelativistic one, characterized by the $S$ - and $D$-state probabilities, i.e., $P(S)=94.23 \% \quad$ and $P(D)=5.77 \%$. The effective nucleon polarization $\left\langle\sigma_{N\left(t_{N}\right)}\right\rangle$ is defined in Eq. (5.16a); it is related to the wave function probabilities according to Eq. (C8) of Appendix C; in the isoscalar deuteron it is the same for proton and neutron; the employed wave function yields the specific values $\left\langle\sigma_{p}\right\rangle=\left\langle\sigma_{n}\right\rangle=0.457$. The proton and neutron components of the front form spin distribution $\vec{s}_{N}\left(\xi t_{N} n_{A}\right)$ are shown in Fig. 1. They are identical. Furthermore, their longitudinal and transverse components are indistinguishable on the scale of the plot.

\section{Trinucleon bound state ${ }^{3} \mathrm{He}$}

The employed relativistic trinucleon wave function is obtained from a nonrelativistic one, computed in Refs. [21,22].
It is characterized by the $S$-, $S^{\prime}$-, and $D$-state probabilities $P(S)=90.36 \%, P\left(S^{\prime}\right)=1.38 \%$, and $P(D)=8.25 \%$. The effective nucleon polarization $\left\langle\sigma_{N\left(t_{N}\right)}\right\rangle$ is defined in Eq. (5.16a) of Sec. V; it is related to the wave function probabilities according to Eq. (5.16b); the employed wave function yields the specific values $\left\langle\sigma_{n}\right\rangle=0.881$ and $\left\langle\sigma_{p}\right\rangle=-0.046$. The neutron component is also in the front form spin distribution of overwhelming importance as Fig. 2 shows; this fact holds true for longitudinal and transverse polarizations. In the front form spin distribution of ${ }^{3} \mathrm{H}$ the proton and neutron change roles. Thus, the technical tools for calculating the ${ }^{3} \mathrm{H}$ spinstructure functions are also available, but no such calculation is done in this paper.

We expected that the Melosh rotation from canonical to front form spin is a sizable relativistic effect, important for the actual magnitude and shape of the front form spin distributions. This expectation did not turn out to be true; leaving the Melosh rotations out, i.e., putting them to unity in Eq. (C7) for the deuteron and in Eq. (6.13) for ${ }^{3} \mathrm{He}$ creates minute changes of less than $0.1 \%$ in the distributions outside zeros; the changes are invisible in the plots. In both cases, deuteron and ${ }^{3} \mathrm{He}$, the front form spin distributions are peaked around $\xi=1 / A$. The approximation

$$
\vec{n}_{A} \cdot \vec{s}_{N \text { app }}\left(\xi t_{N} n_{A}\right)=\delta\left(\xi-\frac{1}{A}\right)\left\langle\sigma_{N\left(t_{N}\right)}\right\rangle
$$

is a simple one; it is independent from the direction $\vec{n}_{A}$ of the nuclear polarization. When calculating the nuclear spinstructure functions $g_{i}^{A}$ later on, it will turn out to also be a reliable one for both nucleonic distributions in both nuclei. It is poorest for the ${ }^{3} \mathrm{He}$ proton distribution in longitudinal polarization whose peaking around $\xi=1 / \mathrm{A}$ is least pronounced. However, the proton contribution to the ${ }^{3} \mathrm{He}$ spin-structure functions will turn out to be small anyhow.

\section{B. Nucleonic spin-structure functions $g_{i}^{N\left(t_{N}\right)}$}

The nuclear spin-structure functions $g_{i}^{A}$ are calculated according to the convolution formulas (5.12). The convolution formulas require a parametrization of the nucleonic spinstructure functions $g_{i}^{N\left(t_{N}\right)}$. We employ the parametrization of Ref. [23]; it is displayed in Figs. 3 and 4. The parametrization of $g_{1}^{N\left(t_{N}\right)}$ in Ref. [23] accounts for the experimental proton data [24] and satisfies the Bjorken sum rule [25] 


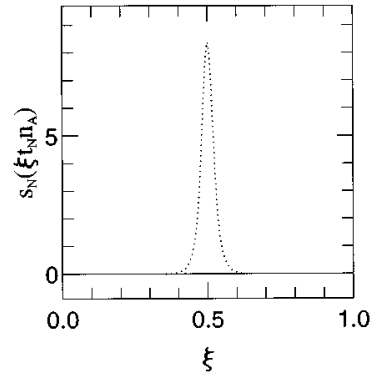

FIG. 1. Components $\vec{n}_{A} \cdot \vec{s}_{N}\left(\xi t_{N} n_{A}\right)$ of the deuteron front form spin distribution as a function of the momentum fraction $\xi$. Neutron and proton components are identical. The longitudinal and transverse contributions, i.e., the contributions for $\vec{n}_{A}=\hat{n}$ and $\vec{n}_{A}=\hat{e}_{1}$, respectively, are indistinguishable on the chosen scale.

$$
\int d x_{N}\left[g_{1}^{p}\left(x_{N}\right)-g_{1}^{n}\left(x_{N}\right)\right]=\frac{1}{6} \frac{g_{A}}{g_{V}},
$$

$g_{A}$ and $g_{V}$ being the axial and vector coupling constants of the nucleon. The parametrization of $g_{2}^{N\left(t_{N}\right)}$ is based on the twist-2 prescription of Ref. [26] which relates both nucleonic spin-structure functions by

$$
g_{2}^{N\left(t_{N}\right)}\left(x_{N}\right)=-g_{1}^{N\left(t_{N}\right)}\left(x_{N}\right)+\int_{x_{N}}^{1} \frac{d x_{N}^{\prime}}{x_{N}^{\prime}} g_{1}^{N\left(t_{N}\right)}\left(x_{N}^{\prime}\right) .
$$

The first data [14] for $g_{2}^{p}$, referring to the momentum transfer range $1 \mathrm{GeV}^{2} \leqslant-Q^{2} \leqslant 10 \mathrm{GeV}^{2}$, are consistent with that prescription, though the experimental precision is still unsatisfactorily poor.

The proton and neutron spin-structure functions $g_{1}^{N\left(t_{N}\right)}$ and $g_{2}^{N\left(t_{N}\right)}$ are of comparable magnitude, though of different sign, in the parametrization of Figs. 3 and 4. This characteristic is decisive for the successful extraction of the neutron spin-structure functions from ${ }^{3} \mathrm{He}$ data. This characteristic
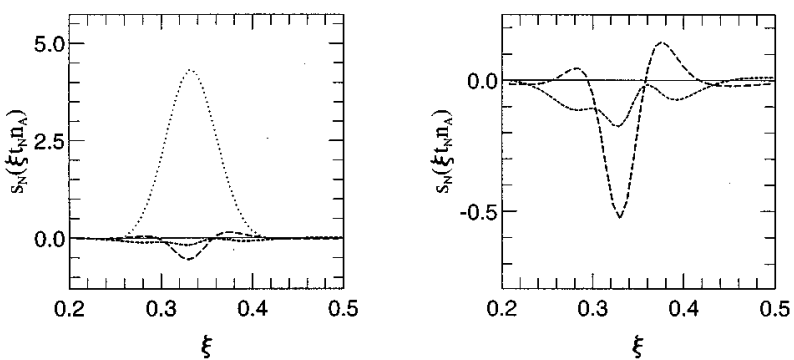

FIG. 2. Component $\vec{n}_{A} \cdot \vec{s}_{N}\left(\xi t_{N} n_{A}\right)$ of the ${ }^{3} \mathrm{He}$ front form spin distribution as function of the momentum fraction $\xi$. Neutron and proton components are shown for longitudinal and transverse polarizations, i.e., for $\vec{n}_{A}=\hat{n}$ and $\vec{n}_{A}=\hat{e}_{1}$, respectively. The neutron components are given by the dotted curve; on the chosen scale of the plot, they coincide for both cases of polarization. The longitudinal (transverse) proton component is displayed as short-dashed (longdashed) curve; the small proton components are also shown in an enlarged scale on the right side of the figure.
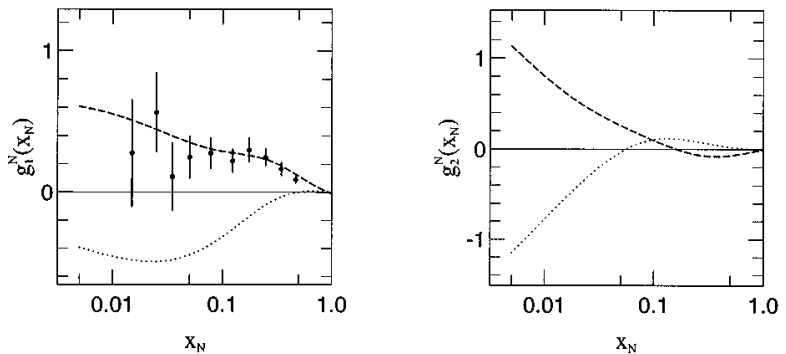

FIG. 3. Nucleonic spin-structure functions $g_{1}^{N\left(t_{N}\right)}$ and $g_{2}^{N\left(t_{N}\right)}$ as functions of the nucleonic Bjorken variable $x_{N}$ in the scaling limit. The neutron spin-structure functions are shown as dotted curves, the proton ones as dashed curves, all in the parametrization of Ref. [23], $g_{2}^{N\left(t_{N}\right)}$ according to the twist-2 prescription of Ref. [26]. The data for the proton spin-structure function $g_{1}^{p}$ are taken from Ref. [24]; only the statistical errors are shown. Older SLAC data for $g_{1}^{p}$ referred to in Ref. [24] are not plotted.

does not hold for the parametrization of $g_{1}^{N\left(t_{N}\right)}$ according to Ref. [27], which seems, however, to be invalidated by the existing proton data [24].

\section{Nuclear spin-structure functions $g_{i}^{A}$}

The deuteron and ${ }^{3} \mathrm{He}$ spin-structure functions $g_{1}^{A}$ and $g_{2}^{A}$ are calculated using the convolution formulas (5.12). The predictions are displayed in Figs. 5 and 6, respectively. They are broken up into proton and neutron contributions. The neutron contribution dominates the ${ }^{3} \mathrm{He}$ spin-structure functions; the proton contribution is insignificant for ${ }^{3} \mathrm{He}$. In contrast, the proton and neutron make contributions of comparable magnitude, but of opposite sign to the deuteron spinstructure functions. Both results are due to the fact that proton and neutron spin structure functions $g_{i}^{N\left(t_{N}\right)}$ are of comparable size. Experimental data on the deuteron and ${ }^{3} \mathrm{He}$ spin-structure function $g_{1}^{A}$ and on the deuteron spinstructure function $g_{2}^{A}$ have recently become available, i.e., Refs. $[12,13,10,11,14]$, respectively.

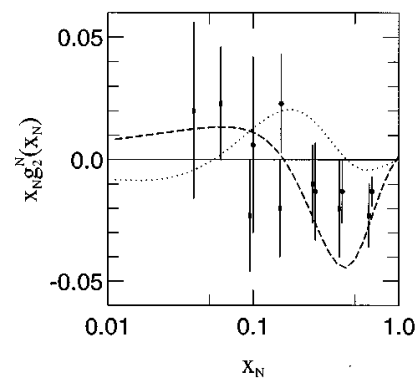

FIG. 4. Weighted nucleonic spin-structure functions $x_{N} g_{2}^{N\left(t_{N}\right)}$ as functions of the nucleonic Bjorken variable $x_{N}$ in the scaling limit. The neutron spin-structure function is shown as dotted curve, the proton one as dashed curve, both in the parametrization of Ref. [23] according to the twist-2 prescription of Ref. [26]. The data for the proton spin-structure function $g_{2}^{p}$ are taken from Ref. [14]; they refer to two distinct scattering angles of the lepton, $\Theta_{e}=4.5^{\circ}$ and $\Theta_{e}=7.0^{\circ}$, indicated by solid dots and solid rectangles, respectively; only the statistical errors are shown. 


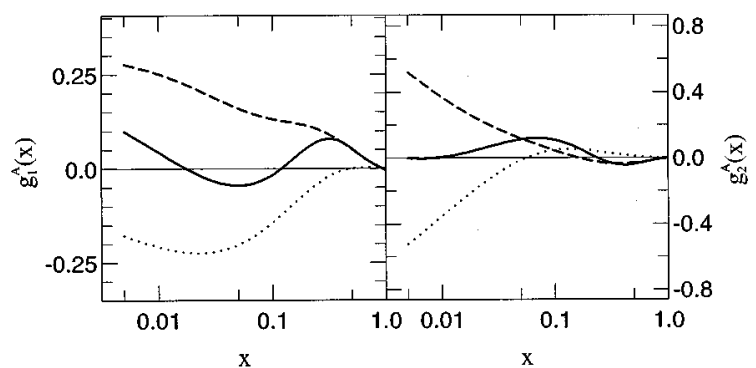

FIG. 5. Deuteron spin-structure functions $g_{1}^{A}$ and $g_{2}^{A}$ as functions of the Bjorken variable $x$. They are derived from the convolution formulas (5.12) using the parametrizations of Fig. 3 for the nucleonic structure functions. The full results are shown as solid curves; the neutron and proton contributions are given by the dotted and dashed curves, respectively.

The deuteron data [12-14] refer to a momentum transfer range $1 \mathrm{GeV}^{2} \leqslant-Q^{2} \leqslant 15 \mathrm{GeV}^{2}$; we assume them to scale and therefore consider the description in scaled approximation developed in this paper as appropriate. Figure $7 \mathrm{com}-$ pares the data with the predictions of Fig. 5. The experimental error bars for the quantity $x g_{1}^{A}$ in Ref. [13] have become quite small indicating that the theoretical spin-structure function changes sign at too large a value for the scaling variable $x$; furthermore its peak is also shifted to too large $x$ values. Thus, as a consequence, also the assumed parametrization of the neutron spin-structure function $g_{1}^{n}$ appears to be slightly inconsistent with the data. In contrast, due to sizable error bars the first data [14] for $x g_{2}^{A}$ cannot be considered a stringent test of the assumed $g_{2}^{N\left(t_{N}\right)}$.

The ${ }^{3} \mathrm{He}$ data $[10,11]$ refer to an average momentum transfer of $-Q^{2}=2 \mathrm{GeV}^{2}$, scaling is not evident; the description in scaled approximation developed in this paper is not quite appropriate. Outside the domain of Bjorken scaling the general convolution formulas for the nuclear spinstructure functions have to be based on the front form spectral function and not on the density which the particular relations (5.12) use; in that case $Q_{N}$ is also not equal to $Q$. The front form spectral function is not calculated in this paper. Nevertheless, the description assuming scaling is able to

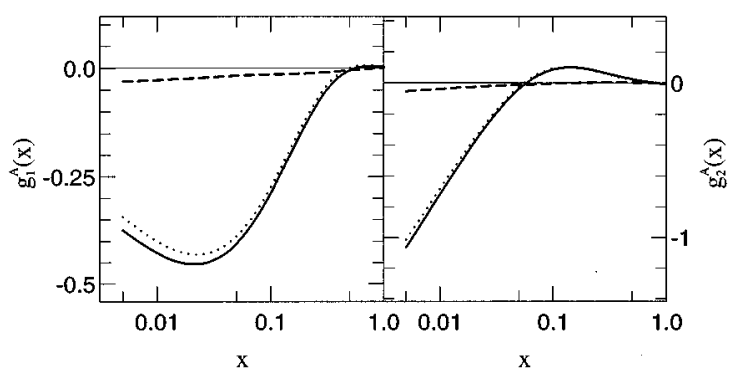

FIG. 6. ${ }^{3} \mathrm{He}$ spin-structure functions $g_{1}^{A}$ and $g_{2}^{A}$ as functions of the Bjorken variable $x$. They are derived from the convolution formulas (5.12) using the parametrizations of Fig. 3 for the nucleonic structure functions. The full results are shown as solid curves; the neutron and proton contributions are given by the dotted and dashed curves, respectively.

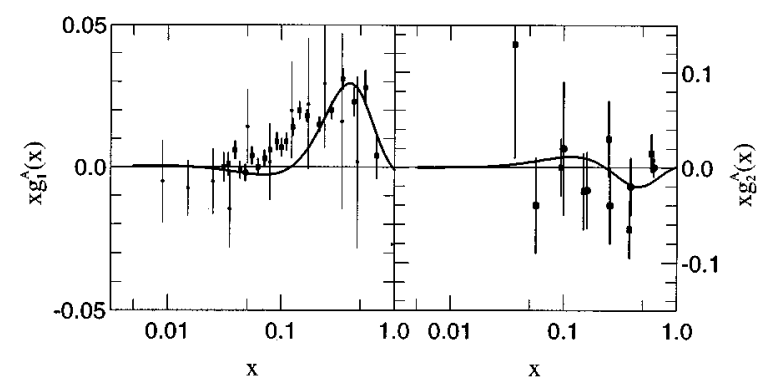

FIG. 7. Weighted deuteron spin-structure functions $x g_{1}^{A}$ and $x g_{2}^{A}$ as functions of the Bjorken variable $x$. The theoretical predictions are derived from the convolution formulas (5.12) using the parametrization of Fig. 3 for the nucleonic structure functions; they are compared to the experimental data for $x g_{1}^{A}$ of [12], indicated by small solid dots, and of [13], indicated by solid rectangles, and to the experimental data for $x g_{2}^{A}$ of [14]; the data for $x g_{2}^{A}$ refer to two distinct scattering angles of the lepton $\Theta_{e}=4.5^{\circ}$ and $\Theta_{e}=7.0^{\circ}$, indicated by solid dots and rectangles, respectively; only the statistical errors are shown.

roughly account for trends in the existing experimental data [11]. Figure 8 compares the theoretical prediction with data.

\section{Unfolding the neutron spin-structure functions from the nuclear ones}

The front form spin distributions $\vec{s}_{N}\left(\xi t_{N} n_{A}\right)$ are approximated in Eq. (7.1) by a $\delta$-function form. This approximation yields approximate nuclear spin-structure functions

$$
g_{i \text { app }}^{A}(x)=\sum_{t_{N}} g_{i}^{N\left(t_{N}\right)}(x)\left\langle\sigma_{N\left(t_{N}\right)}\right\rangle
$$

in turn, i.e., very simple relations between nuclear and nucleonic spin-structure functions. Approximation (7.4) accounts for the full calculation quite reliably. For the deuteron and for ${ }^{3} \mathrm{He}$ the differences in $g_{1}^{A}$ and $g_{2}^{A}$ between the approximated forms (7.4) and the full convolution formulas (5.12) are not distinguishable in plots and are therefore not displayed-with one rather inconsequential exception: As expected from the discussion of the ${ }^{3} \mathrm{He}$ proton distribution, the approximated proton contributions to $g_{i}^{A}$ show some minor deviations; they are given in Fig. 9. The transverse component of the proton spin distribution in ${ }^{3} \mathrm{He}$ is compara-

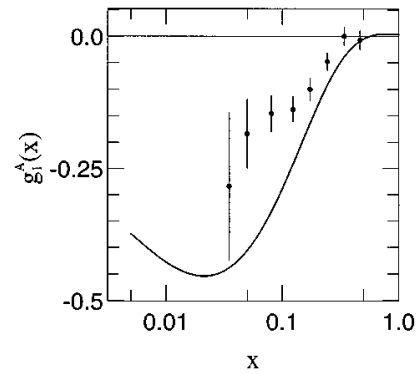

FIG. 8. ${ }^{3} \mathrm{He}$ spin-structure function $g_{1}^{A}$ as function of the Bjorken variable $x$. The theoretical prediction is derived from the convolution formula (5.12a) using the parametrization of Fig. 3 for the nucleonic structure functions; it is compared to the experimental data of Ref. [11]; for the data only the statistical errors are shown. 


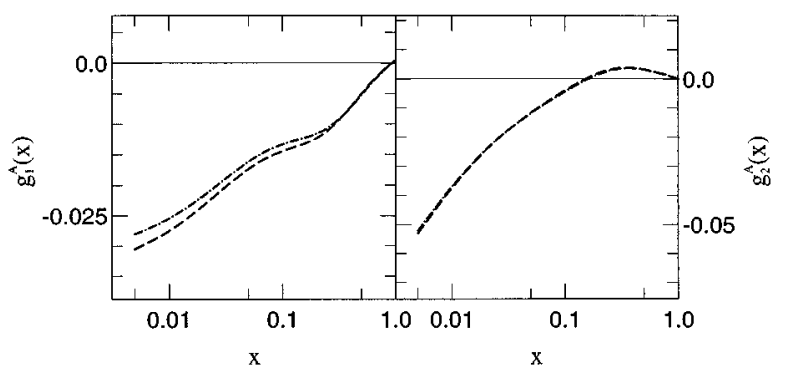

FIG. 9. ${ }^{3} \mathrm{He}$ spin-structure functions $g_{1}^{A}$ and $g_{2}^{A}$ as functions of the Bjorken variable $x$. The validity of the approximation (7.4) is checked. Only the small proton contributions are shown. Compared with Fig. 6 the resolution of the plots is increased. The proton contributions are (i) derived in a proper calculation according to the convolution formulae of Eqs. (5.12) and (ii) estimated according to the approximation (7.4); the dashed curves refer to the proper calculation, the dot-dashed ones to the approximation. The corresponding neutron contributions and the full spin-structure functions are not shown, since both results are indistinguishable in plots with the chosen resolution.

tively better approximated by Eq. (7.1) than the longitudinal one as the different pronounciation of peaks around $\xi=1 / \mathrm{A}$ in Fig. 2 shows. According to Eqs. (5.12) the spin-structure function $g_{1}^{A}$ depends on the longitudinal and the spinstructure function $g_{2}^{A}$ on the transverse component of the front form spin distribution $\vec{s}_{N}\left(\xi t_{N} n_{A}\right)$. The differences between the approximated form (7.4) and the full convolution formula $(5.12 \mathrm{~b})$ for $g_{2}^{A}$ are therefore smaller than the corresponding differences for $g_{1}^{A}$. This fact is bourne out in Fig. 9 for the proton contributions. We now turn to unfolding the neutron spin-structure functions from the nuclear ones.

First, we assume that the convolution formulas (5.12) are exact. However, we would like to use its approximated form (7.4) in order to extract the neutron spin-structure functions $g_{i}^{n}$ from the experimental data

$$
g_{i}^{n}\left(x_{N}\right)=\left.\left[g_{i}^{A}(x)-g_{1}^{p}(x)\left\langle\sigma_{p}\right\rangle\right] \frac{1}{\left\langle\sigma_{n}\right\rangle}\right|_{x=x_{N}} .
$$

An example is given in Fig. 10. Even if the convolution formula (5.12a) were exact and were well described by Eq. (7.4), the extraction (7.5) of the neutron spin-structure functions meets three distinct types of errors, i.e.,

$$
\begin{aligned}
\Delta g_{i}^{n}\left(x_{N}\right)= & \left(\Delta g_{i}^{A}\left(x_{N}\right) \frac{1}{\left\langle\sigma_{n}\right\rangle}+\Delta g_{i}^{p}\left(x_{N}\right)\left|\frac{\left\langle\sigma_{p}\right\rangle}{\left\langle\sigma_{n}\right\rangle}\right|\right) \\
& +\Delta\left\langle\sigma_{p}\right\rangle\left|\frac{g_{i}^{p}\left(x_{N}\right)}{\left\langle\sigma_{n}\right\rangle}\right|+\Delta\left\langle\sigma_{n}\right\rangle \mid\left(g_{i}^{A}\left(x_{N}\right)\right. \\
& \left.-g_{i}^{p}\left(x_{N}\right)\left\langle\sigma_{p}\right\rangle\right) \frac{1}{\left\langle\sigma_{n}\right\rangle^{2}} \mid+\Delta_{\text {sys }} g_{i}^{n}\left(x_{N}\right),
\end{aligned}
$$

which we add up linearly for convenience.

(1) There is a systematic error $\Delta_{\text {sys }} g_{i}^{n}\left(x_{N}\right)$ due to the fact that the extraction (7.5) is derived from the approximate con-

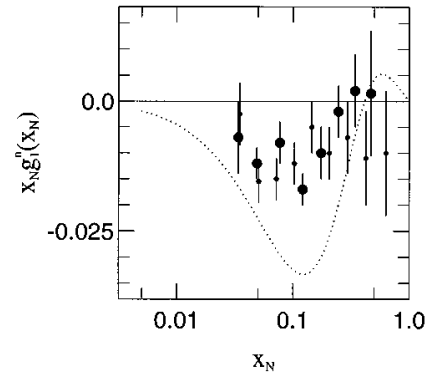

FIG. 10. Weighted neutron spin-structure function $x_{N} g_{1}^{n}$ as a function of the Bjorken variable $x_{N}$. The neutron spin-structure function assumed in Fig. 3 is compared to the ones extracted from the ${ }^{3} \mathrm{He}$ data [10], indicated by big solid dots, and from the deuteron data [13], indicated by solid dots, according to Eq. (7.5). The model dependence of the extraction due to the nonuniqueness of the deuteron wave function is not folded into the experimental error; it is small compared with the existing errors in the experimental data.

volution formula (7.4). When starting out from the parametrization of the nucleonic spin-structure functions in Fig. 3, calculating the nuclear ones without approximation according to Eq. (5.12) and then recovering $g_{i}^{n}$ according to the approximate relation (7.5), differences arise which are not resolvable in plots and are therefore not displayed. This systematic error therefore appears to be minor.

(2) The experimental errors of $g_{i}^{A}$ and $g_{i}^{p}$, i.e., $\Delta g_{i}^{A}$ and $\Delta g_{i}^{p}$, pollute the extracted neutron spin-structure functions. The errors on $\Delta g_{i}^{A}$ in the existing experiments are still large. For the deuteron the error in the existing proton data contributes to the error in the extraction quite significantly, since $\Delta g_{i}^{p}\left|\left\langle\sigma_{p}\right\rangle /\left\langle\sigma_{n}\right\rangle\right|=\Delta g_{i}^{p}$; in contrast the same error has a very small weight for the extraction from ${ }^{3} \mathrm{He}$ data, since $\Delta g_{i}^{p}\left|\left\langle\sigma_{p}\right\rangle /\left\langle\sigma_{n}\right\rangle\right| \simeq 0.05 \Delta g_{i}^{p}$.

(3) The nucleonic contributions $\left\langle\sigma_{N\left(t_{N}\right)}\right\rangle$ in the extraction (7.5) are model dependent, though they lie within rather small limits for realistic bound state wave functions, i.e., for the deuteron $0.45 \leqslant\left\langle\sigma_{p, n}\right\rangle \leqslant 0.47$ and for ${ }^{3} \mathrm{He} \quad$ [1] $0.85 \leqslant\left\langle\sigma_{n}\right\rangle \leqslant 0.90$ and $-0.06 \leqslant\left\langle\sigma_{p}\right\rangle \leqslant-0.04$. The resulting spread of theoretical results is displayed in Fig. 11 for ${ }^{3} \mathrm{He}$. Given the errors in the present experimental data, the model dependence is not significant yet.

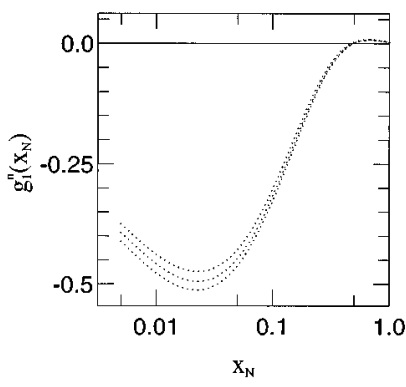

FIG. 11. Neutron spin-structure function $g_{1}^{n}$ as a function of the Bjorken variable $x_{N}$. The neutron spin-structure function assumed according to Fig. 3 is shown as the middle curve and compared with the ones extracted from the calculated ${ }^{3} \mathrm{He}$ spin-structure function $g_{1}^{A}$ according to Eq. (7.5). The upper and lower curves give the bandwidth of the theoretical uncertainty due to the model dependence [1] of the trinucleon bound state wave function. 
Second, the convolution formulas (5.12) may be conceptually wrong. There may be a multitude of effects, e.g., a medium dependence of the nucleonic structure functions, a binding correction, or other contributing non-nucleonic nuclear constituents, which invalidate the convolution formulas (5.12) and which also contribute to $\Delta_{\text {sys }} g_{i}^{n}\left(x_{N}\right)$. Those effects were discussed in the context of the spin-independent structure function $F_{2}^{A}$ [18]. In the next subsection we worry about corresponding consequences for the spin-structure functions $g_{i}^{A}$ and their bearing on extracting neutron spinstructure functions. Anticipating that also those corrections will turn out to be small, we conclude:

The approximate relation (7.5) can reliably be used to extract the neutron spin-stucture functions $g_{i}^{n}$ of the neutron from deuteron and ${ }^{3} \mathrm{He}$ data. Figure 10 uses the ${ }^{3} \mathrm{He}$ data of Ref. [10] and the deuteron data of Ref. [13]. When the data from all experiments, presently being performed or being in the planning stage, will be in, the parametrization of the neutron spin structure functions $g_{i}^{n}$, shown in Fig. 3, may have to be revised.

\section{E. Is there an EMC effect in the ${ }^{3} \mathrm{He}$ spin structure function $g_{1}^{A}$ ?}

In the case of unpolarized deep-inelastic lepton scattering a deviation between the nucleonic structure function of a free nucleon, i.e., $F_{2}^{N\left(t_{N}\right)}$, and of a bound nucleon, i.e., $F_{2}^{A} / A$, is indeed observed and called the EMC effect. There is a diversity of theoretical mechanisms [28] able to account for that observation. For example, the decrease of the EMC ratio

$$
R_{\mathrm{EMC}}(x):=\frac{F_{2}^{A}(x)}{2 F_{2}^{p}(x)+F_{2}^{n}(x)}
$$

at intermediate values of the Bjorken variable $x$, defined here for ${ }^{3} \mathrm{He}$, is often attributed to a medium correction of the nucleonic structure function, which may be realized in a theoretical description either by modifying the nucleonic front form momentum distribution due to nuclear binding or by an additional meson contribution to the nuclear structure function, the pion being the most important non-nucleonic nuclear constituent; Ref. [18], which this paper extends, used the latter mechanism. Both mechanisms yield comparable effects for $F_{2}^{A}$ at intermediate $x$; they affect, however, the spin-structure function $g_{1}^{A}$ quite differently: Whereas a binding modification of the spin-averaged momentum distribution will also yield a corresponding modification of the front form spin distribution $\vec{s}_{N}\left(\xi t_{N} n_{A}\right)$ and will directly change the predictions for the nuclear spin-structure function $g_{1}^{A}$, we do not see any simple mechanism for the spinless pions to contribute to $g_{1}^{A}$.

In one recipe attempting to account for nuclear binding [29], the nucleonic plus momentum $p_{N}^{+}$is shifted to $p_{N}^{+}+\epsilon$ due to binding, $\epsilon$ standing for an averaged binding energy, often taken to be about $-30 \mathrm{MeV}$. Indeed, such a simple shift yields the decrease in the ordinary EMC ratio $R_{\text {EMC }}(x)$ at intermediate Bjorken variables $x$. Using the convolution formula

$$
F_{2 \text { binding }}^{A}(x)=\sum_{t_{N}} \int d \xi F_{2}^{N\left(t_{N}\right)}\left(\frac{x}{A \xi}\right) f_{N \text { binding }}\left(\xi t_{N}\right)
$$

with the spin-averaged front form momentum distribution

$$
\begin{aligned}
f_{N \text { binding }}\left(\xi t_{N}\right):= & \int d^{3} \mathbf{p}_{N} \delta\left(\xi-\frac{p_{N}^{+}+\epsilon}{P_{A}^{+}}\right) \\
& \times \operatorname{Tr}\left[\rho\left(\mathbf{p}_{N} t_{N}\right) \rho_{A}\left(n_{A} P_{A}\right)\right],
\end{aligned}
$$

into which binding is built in, the EMC effect in the EMC ratio $R_{\mathrm{EMC}}$ can by and large be accounted for. Assuming the same momentum shift in the nucleonic plus momentum for the present discussion of the ${ }^{3} \mathrm{He}$ spin-structure function $g_{1}^{A}$, the front form spin distribution $\vec{s}_{N}\left(\xi t_{N} n_{A}\right)$ gets the correspondingly changed modification and becomes

$$
\begin{aligned}
& \vec{s}_{N \text { binding }}\left(\xi t_{N} n_{A}\right):= d^{3} \mathbf{p}_{N} \delta\left(\xi-\frac{p_{N}^{+}+\epsilon}{P_{A}^{+}}\right) \\
& \times \operatorname{Tr}\left[\vec{\sigma}_{N} \rho\left(\mathbf{p}_{N} t_{N}\right) \rho_{A}\left(n_{A} P_{A}\right)\right], \\
& \vec{s}_{N \text { binding }}\left(\xi t_{N} n_{A}\right)=\vec{s}_{N}\left[\left(\xi-\frac{\epsilon}{m_{A}}\right) t_{N} n_{A}\right]
\end{aligned}
$$

The shift in the nucleonic plus momentum is only introduced in the $\delta$ function which contains the front form momentum fraction $p_{N}^{+} / P_{A}^{+}$. The front form spin distribution $\vec{s}_{N \text { binding }}\left(\xi t_{N} n_{A}\right)$ still satisfies the sum rule (5.15) almost unchanged: Compared with the original definition (5.13) its dependence on $\xi$ is shifted, but since its nonvanishing values are centered around 1/A according to Figs. 1 and 2, the integration of $\vec{n}_{A} \cdot \vec{s}_{N \text { binding }}\left(\xi t_{N} n_{A}\right)$ over $\xi$ from 0 to 1 still collects the complete domain in which the front form spin distribution is nonzero. The changed front form spin distribution in Eq. (5.12a) yields the modified ${ }^{3} \mathrm{He}$ spinstructure function $g_{1 \text { binding }}^{A}$ according to

$g_{1 \text { binding }}^{A}(x)=\left.\sum_{t_{N}} \int \frac{d \xi}{A \xi} g_{1}^{N\left(t_{N}\right)}\left(\frac{x}{A \xi}\right) \vec{n}_{A} \cdot \vec{s}_{N \text { binding }}\left(\xi t_{N} n_{A}\right)\right|_{n_{A} \|} \|$

When using that modified ${ }^{3} \mathrm{He}$ spin-structure function

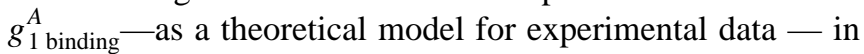
the extraction procedure (7.5) for the neutron spin-structure function $g_{1}^{n}$, no significantly increased deviation occurs between the neutron spin-structure function extracted and the one assumed for calculating $g_{1}^{A}$ binding according to Eq. (7.10). In fact, compared with the errors $\Delta g_{1}^{n}$ discussed in the last subsection the deviation is increased by less than $1 \%$; the deviation would not be discernable in a plot with the resolution of Fig. 10. Thus, a possible binding correction in the ${ }^{3} \mathrm{He}$ spin-structure function $g_{1}^{A}$, as introduced in this subsec- 

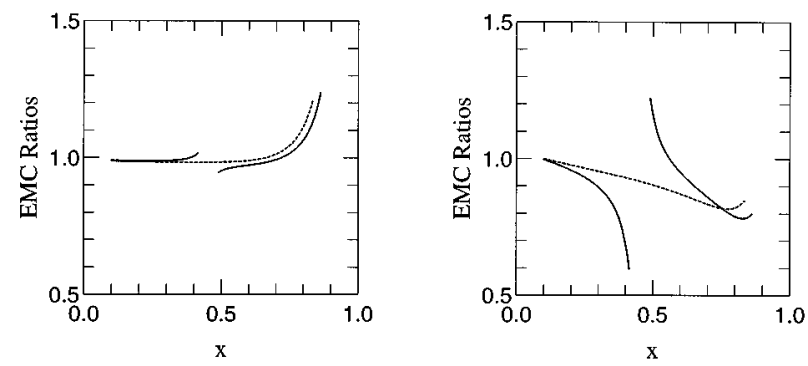

FIG. 12. EMC ratios $S_{\mathrm{EMC}}(x)$ and $R_{\mathrm{EMC}}(x)$. The spin ratio $S_{\mathrm{EMC}}(x)$ of Eq. (7.11) is given as solid curve and compared with the ordinary EMC ratio $R_{\mathrm{EMC}}(x)$ of Eq. (7.7) given as a dashed curve. In the right figure the nuclear structure functions are used in the forms $g_{1 \text { binding }}^{A}(x)$ and $F_{2 \text { binding }}^{A}(x)$ according to Eqs. (7.10) and (7.8a). The left figure shows the same ratios but without the inclusion of binding effects; there, the nuclear structure functions are used in the forms $g_{1}^{A}(x)$ and $F_{2}^{A}(x)$ according to Eqs. (5.12a) and (7.8a) with $\epsilon=0$.

tion, does not invalidate the extraction of the neutron spinstructure function according to Eq. (7.5). However, an EMC effect can arise in the ratio

$$
S_{\mathrm{EMC}}(x):=\frac{g_{1}^{A}(x)}{g_{1}^{p}(x)\left\langle\sigma_{p}\right\rangle+g_{1}^{n}(x)\left\langle\sigma_{n}\right\rangle},
$$

$S_{\text {EMC }}$ being a much more sensitive quantity. This fact is documented in Fig. 12 which shows this ratio with and without the inclusion of a binding correction according to Eq. (7.10) in the calculation of the ${ }^{3} \mathrm{He}$ spin-structure function $g_{1}^{A}$. The ratio $S_{\mathrm{EMC}}$ exhibits the same decrease at intermediate Bjorken variables $x$ as the ordinary EMC ratio $R_{\mathrm{EMC}}$ of Eq. (7.7) does. The trend is clearly observable in $S_{\mathrm{EMC}}$, though its smooth dependence on the Bjorken variable $x$ is disturbed by the zero in the denominator of the ratio in definition (7.11); the definition may therefore be considered unfortunate.

\section{F. Relation to instant form dynamics}

Reference [3] describes polarized inelastic lepton scattering from ${ }^{3} \mathrm{He}$ noncovariantly in PWIA. It assumes instant form dynamics. Though the formalism is employed there for a description of quasielastic scattering, it is general and may formally be applied to the deep-inelastic regime of the Bjorken limit as well. In this subsection we explore a description of deep-inelastic scattering based on Eqs. (3.11a)(3.11d) of Ref. [3]. However, the exploration is only a matter of curiosity: The boosts of instant form dynamics are interaction dependent for a composite system. The assumption of PWIA that the nuclear current is built from single-nucleon contributions cannot be made consistently. Thus, front form dynamics remains the superior form of description for deepinelastic scattering.

The description of Ref. [3] in terms of instant form dynamics made the same conceptual distinction between the momentum transfer to the nucleus $Q$ and to a nucleon $Q_{N}$, to a nucleon bound in the nucleus though assumed to be on mass shell, as this paper does, i.e., $Q_{N} \neq Q$ due to their difference in energy transfer. The difference between the en- ergy transfers to the struck nucleon $Q_{N}$ and to the nucleus $Q$ is relatively small for deep-inelastic scattering, i.e., $\Delta:=Q^{0}-Q_{N}^{0},|\Delta| \ll Q^{0}$. Nevertheless, its effect on $Q_{N}^{2}$

$$
Q_{N}^{2}=Q^{2}-2 Q^{0} \Delta+\Delta^{2}
$$

can be sizable. One finds for the ratio of the scaling variables $x$ and $x_{N}$

$$
\begin{aligned}
& \frac{x}{x_{N}}=\frac{Q^{2}}{2 P_{A} \cdot Q} A \frac{2 p_{N} \cdot Q_{N}}{Q_{N}^{2}}, \\
& \frac{x}{x_{N}} \simeq \frac{1}{1+\Delta / m_{N} x} \frac{A p_{N} \cdot Q}{P_{A} \cdot Q},
\end{aligned}
$$

with

$$
p_{N} \cdot Q_{N} \simeq p_{N} \cdot Q
$$

The ratio $x / x_{N}$ is only at moderate values of $x$ close to $A p_{N}^{+} / P_{A}^{+}$for all relevant nucleon momenta $p_{N}$ at which the spectral function gives significant contributions. Comparing the instant form and the front form convolution formulas we therefore have to expect conflicting results for small values of the Bjorken variable $x$.

The noncovariant result for $g_{1}^{A}\left(x, Q^{2}\right)=\left(Q \cdot P_{A} /\right.$ $\left.A m_{A}\right) G_{1}^{A}\left(Q^{2}, P_{A} \cdot Q / m_{A}\right)$ is obtained from Eq. (3.11c) of Ref. [3] as function of $x$ and $Q^{2}$. The only modification with respect to the quasielastic results is the replacement of the nucleonic structure functions by the corresponding deepinelastic ones according to

$$
\begin{gathered}
G_{1}^{N\left(t_{N}\right)}\left(Q_{N}^{2}, p_{N} \cdot Q_{N} / m_{N}\right)=\lim _{B j} \frac{m_{N}}{p_{N} \cdot Q_{N}} g_{1}^{N\left(t_{N}\right)}\left(x_{N}, Q_{N}^{2}\right), \\
G_{2}^{N\left(t_{N}\right)}\left(Q_{N}^{2}, p_{N} \cdot Q_{N} / m_{N}\right)=\lim _{B j} \frac{m_{N}^{3}}{\left(p_{N} \cdot Q_{N}\right)^{2}} g_{2}^{N\left(t_{N}\right)}\left(x_{N}, Q_{N}^{2}\right) .
\end{gathered}
$$

For practical calculations the parametrizations of $g_{i}^{N\left(t_{N}\right)}$ given in Sec. VII B are used; they do not carry a dependence on $Q_{N}^{2}$; in contrast, the nucleonic structure functions $G_{i}^{N\left(t_{N}\right)}$ will remain dependent on $Q_{N}^{2}$.

The solid curve of Fig. 13 shows the spin-structure function $g_{1}^{A}\left(x, Q^{2}\right)$ derived from Eq. (3.11c) of Ref. [3]. The four-momentum transfer has been chosen to be $-Q^{2}=10$ $\mathrm{GeV}^{2}$. However, no significant change of the results is seen for any value larger than $-Q^{2} \geqslant 1 \mathrm{GeV}^{2}$. Thus, theoretically, scaling is observed for $-Q^{2} \gg 1 \mathrm{GeV}^{2}$ provided it is assumed for nucleons. This fact may be taken as one justification for assuming scaling in the description of the data of Refs. [10,11], collected for an average momentum transfer $Q^{2}=2 \mathrm{GeV}^{2}$. However, Fig. 13 also proves that the conceptually required difference between the momentum transfer $Q$ to the nucleus and $Q_{N}$ to the nucleon matters. The unjustified recipe $Q_{N}=Q$ makes the results very close to the prediction based on front form dynamics. References [30-34] employ this recipe $Q_{N}=Q$. We claim that the approximated form of Eq. (7.4) for $g_{i}^{A}$, used for the interpretation of ex- 


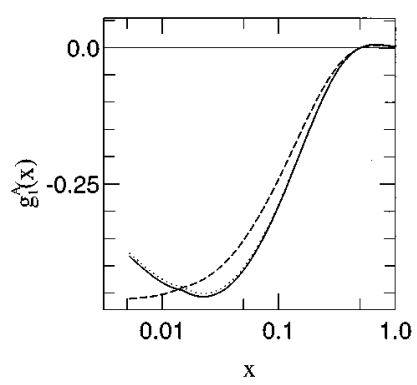

FIG. 13. ${ }^{3} \mathrm{He}$ spin-structure function $g_{1}^{A}$ as function of the Bjorken variable $x$. The result derived from the convolution formula (3.11c) of Ref. [3] using a noncovariant approach under the assumption of instant form dynamics is given as a dashed curve. In a second calculation based on the same formalism we have explicitly set $Q_{N}=Q$; that approximation cannot be justified theoretically in instant form dynamics; the result is presented as dotted curve. For comparison the full calculation of Eq. (5.12a) is shown as a solid curve.

perimental data, is well supported by front form dynamics; in contrast, its derivation based on instant form dynamics is only poorly justified.

\section{CONCLUSION}

The convolution formulas which relate the structure functions for deep-inelastic scattering to the properties of constituent particles of the target nucleus depend on the assumption of one-body current operators and on the covariance of the current tensor of the bound constituents. Consistency problems and ambiguities are due to the fact that one-body currents cannot be covariant under all Lorentz transformations in a system of bound particles.

This paper presents a detailed derivation of nuclear spindependent structure functions for deep-inelastic lepton scattering and applies them to the two- and three-nucleon bound states. Front form dynamics is used for which the covariance under the kinematic subgroup is sufficient to derive convolution formulas: The restriction to one-nucleon currents is possible without inconsistencies. This is why we like the description in terms of front form dynamics. The previous calculation of Ref. [30] is closest in spirit to this paper. In contrast the noncovariant approach using instant form dynamics as discussed in Refs. [3,31-34] cannot be consistently extended to the Bjorken limit in this sense. Our investigation of relativistic effects in the spin-dependent structure functions for the deep-inelastic regime is novel; it extends the work of Ref. [18] to polarization. The numerical results show that relativistic effects due to the proper treatment of spin are small and negligible compared to larger effects arising from the conceptual difference between front form and instant form predictions and arising from the model dependence of the ground state wave functions. These findings are equally valid for the two- and three-nucleon systems.

Front form dynamics allows the formulation of convolution formulas with a comparatively simple structure, i.e., the nuclear spin-structure functions $g_{1}^{A}$ and $g_{2}^{A}$ are separately determined by the convolution of the front form spin distribution with the nucleonic spin-structure functions $g_{1}^{N\left(t_{N}\right)}$ and
$g_{2}^{N\left(t_{N}\right)}$, respectively. In deep-inelastic scattering the simple approximation (7.4) for the convolution formulas is motivated and proven to be reliable. References [1,32] already related the nuclear spin-structure functions to the nucleonic spin-structure functions weighted by integral properties of the nuclear wave function. But they were unable to justify that relation rigorously. The present approach gives the missing proper justification. This finding is important for the interpretation of the experimental data with respect to the analysis of nuclear effects.

\section{ACKNOWLEDGMENTS}

The work was started together with F. Coester who accompanied the authors for a long time with his expertise and encouragement; the authors are indebted to him; the authors owe their slowly acquired insight into the intricacies of front form dynamics entirely to him, their still limited understanding of it is their own fault. The authors are grateful to R. Milner for discussions on the HERMES project. The work was supported in part by the Deutsche Forschungsgemeinschaft (DFG) under Contract No. Sa 247/11-2.

\section{APPENDIX A: DEUTERON DENSITY OPERATOR}

The full density operator $\rho_{A}\left(n_{A}\right)$ commutes with the momentum operator $P$. Its projection $\rho_{A}\left(n_{A} P_{A}\right)$, i.e.,

$$
\left\langle\mathbf{P}_{A} \lambda_{A}^{\prime}\left|\rho_{A}\left(n_{A}\right)\right| \mathbf{P}_{A} \lambda_{A}\right\rangle=\left\langle\lambda_{A}^{\prime}\left|\rho_{A}\left(n_{A} P_{A}\right)\right| \lambda_{A}\right\rangle,
$$

onto the Hilbert sector of momentum eigenstates with eigenvalue $\mathbf{P}_{A}$ is needed in Eq. (3.2). The density operator of a spin-1 target is parametrized in the form

$$
\rho_{A}\left(n_{A} P_{A}\right)=\frac{1}{3}\left[1-\frac{3}{2 m_{A}} n_{A} \cdot W+\frac{3}{m_{A}^{2}} t_{A \mu \nu} T^{\mu \nu}(W)\right]
$$

with the Lorentz tensor $T^{\mu \nu}(W)$ defined by

$$
T^{\mu \nu}(W):=\frac{W^{\mu} W^{\nu}+W^{\nu} W^{\mu}}{2}+\frac{1}{3}\left(\frac{P_{A}^{\mu} P_{A}^{\nu}}{P_{A}^{2}}-g^{\mu \nu}\right) W^{2}
$$

The density operator is at most quadratic in the PauliLubanski vector $W$. The vector $n_{A}$ and the tensor $t_{A}$ describe the polarization of the target. The parametrization (A2) satisfies the required constraints. The Pauli-Lubanski vector $W$ and the tensor operator $T^{\mu \nu}(W)$ are traceless, i.e., $\operatorname{Tr}[W]=0$ and $\operatorname{Tr}\left[T^{\mu \nu}(W)\right]=0$. In general, the polarization vector $n_{A}$ and the polarization tensor $t_{A}$ are independent. However, when considering a quantum mechanically pure state as we do, then $n_{A}$ is the direction according to which its polarization is defined; in this case the polarization vector $n_{A}$ and the polarization tensor $t_{A}$ are connected by

$$
t_{A}^{\mu \nu}=\frac{3}{2}\left[n_{A}^{\mu} n_{A}^{\nu}-\frac{1}{3}\left(\frac{P_{A}^{\mu} P_{A}^{\nu}}{P_{A}^{2}}-g^{\mu \nu}\right)\right],
$$


besides Eq. (A4) the general constraints $n_{A}^{2}=-1$ and $n_{A} \cdot P_{A}=0$ then hold for the polarization vector $n_{A}$.

Matrix elements of the density operator of any momentum $P_{A}$ are related to those of the rest frame, i.e.,

$$
\begin{aligned}
\left\langle\lambda_{A}^{\prime}\left|\rho_{A}\left(n_{A} P_{A}\right)\right| \lambda_{A}\right\rangle= & \left\langle\lambda_{A}^{\prime}\right| \frac{1}{3}\left[1+\frac{3}{2} \hat{n}_{R} \cdot \vec{S}_{A}\right. \\
& \left.+3 \sum_{i j} t_{R i j} T_{A}^{i j}\left(S_{A}\right)\right]\left|\lambda_{A}\right\rangle .
\end{aligned}
$$

For Eq. (A5) the identities

$$
\begin{aligned}
& \frac{W^{\mu}}{m_{A}}\left|\mathbf{P}_{A} \lambda_{A}\right\rangle=\sqrt{\frac{m_{A}}{P_{A}^{+}}} U\left[L_{f}\left(\frac{P_{A}}{m_{A}}\right)\right] \\
& \times\left[L_{f}\left(\frac{P_{A}}{m_{A}}\right)_{\rho}^{\mu}\left(0, \vec{S}_{A}\right)^{\rho}\right]\left|\mathbf{P}_{R} \lambda_{A}\right\rangle \\
& n_{A}^{\mu}=L_{f}\left(\frac{P_{A}}{m_{A}}\right)_{\rho}^{\mu}\left(0, \hat{n}_{R}\right)^{\rho}, \\
& \frac{T^{\mu \nu}(W)}{m_{A}^{2}}\left|\mathbf{P}_{A} \lambda_{A}\right\rangle=\sqrt{\frac{m_{A}}{P_{A}^{+}}} U\left[L_{f}\left(\frac{P_{A}}{m_{A}}\right)\right] \\
& \times\left[L_{f}\left(\frac{P_{A}}{m_{A}}\right)_{i}^{\mu} L_{f}\left(\frac{P_{A}}{m_{A}}\right)_{j}^{\nu} T_{A}^{i j}\left(S_{A}\right)\right]\left|\mathbf{P}_{R} \lambda_{A}\right\rangle,
\end{aligned}
$$

$$
t_{A}^{\mu \nu}=L_{f}\left(\frac{P_{A}}{m_{A}}\right)^{\mu} L_{f}\left(\frac{P_{A}}{m_{A}}\right)_{j}^{\nu} t_{R}^{i j}
$$

are used. In the form (A5) for the target rest frame it is obvious that the density operator has only nine independent elements as appropriate for a spin-1 particle, one arising from the identity operator, three from the vector operator $\vec{S}_{A}$, and five from the tensor operator $T_{A}\left(S_{A}\right)$. The Cartesian rest frame tensor $T_{A}^{i j}\left(S_{A}\right)$,

$$
T_{A}^{i j}\left(S_{A}\right):=\frac{S_{A}^{i} S_{A}^{j}+S_{A}^{j} S_{A}^{i}}{2}-\frac{1}{3} \delta^{i j} \vec{S}_{A}^{2},
$$

is symmetric, i.e., $T_{A}^{i j}\left(S_{A}\right)=T_{A}^{j i}\left(S_{A}\right)$, and with respect to the tensor lables traceless, i.e., $\Sigma_{i} T_{A}^{i i}\left(S_{A}\right)=0$; thus, the tensor part of the density operator can equivalently be written as a five-component tensor $T_{A}^{[2]}\left(S_{A}\right)$ of second rank-in standard relation to its Cartesian representation (A7). The constraints on the density operator $\rho_{A}\left(n_{A} P_{A}\right)$, from which, for example, condition (A4) results, are best proven in the target rest frame, and their validity then carries over to any system by Lorentz covariance.

\section{APPENDIX B: DEUTERON CURRENT TENSOR}

The current tensor $W_{A}^{\mu \nu}\left(Q, P_{A}\right)$ is Hermitian and conserved, it preserves parity and time-reversal invariance. Its Lorentz structure is built from the three four-vectors $Q, P_{A}$, and $W$. The current tensor has the general form

$$
\begin{aligned}
W_{A}^{\mu \nu}\left(Q, P_{A}\right)= & {\left[\frac{Q^{\mu} Q^{\nu}}{Q^{2}}-g^{\mu \nu}\right]\left(F_{1}^{A}\left(x, Q^{2}\right)+\frac{Q \cdot T(W) \cdot Q}{Q^{2} m_{A}^{2}} b_{1}^{A}\left(x, Q^{2}\right)\right) \frac{A}{m_{A}}+\frac{\widetilde{P}_{A}^{\mu} \widetilde{P}_{A}^{\nu}}{Q \cdot P_{A}}\left(F_{2}^{A}\left(x, Q^{2}\right)+\frac{Q \cdot T(W) \cdot Q}{Q^{2} m_{A}^{2}} b_{2}^{A}\left(x, Q^{2}\right)\right) \frac{1}{m_{A}} } \\
& +G^{\mu \nu}(W) b_{3}^{A}\left(x, Q^{2}\right) \frac{1}{m_{A}}+H^{\mu \nu}(W) b_{4}^{A}\left(x, Q^{2}\right) \frac{1}{m_{A}}+i \frac{1}{j} \frac{\epsilon^{\mu \nu \alpha \beta}}{Q \cdot P_{A}} \\
& \times Q_{\alpha}\left[W_{\beta} g_{1}^{A}\left(x, Q^{2}\right)+\left(W_{\beta}-\frac{Q \cdot W}{Q \cdot P_{A}} P_{A \beta}\right) g_{2}^{A}\left(x, Q^{2}\right)\right] \frac{A}{m_{A}}
\end{aligned}
$$

with

$$
\begin{gathered}
\widetilde{P}_{A}^{\mu}:=P_{A}^{\mu}-\frac{Q \cdot P_{A}}{Q^{2}} Q^{\mu}, \\
x:=-\frac{A Q^{2}}{2 Q \cdot P_{A}},
\end{gathered}
$$

$x$ being the Bjorken scaling variable. The dependence of the current tensor operator on the Pauli-Lubanski vector $W$ is at most quadratic for a spin-1 target, $j=1$ being the spin quantum number. Besides the standard basic tensor forms used for a spin- $\frac{1}{2}$ case the two additional ones $\left[\widetilde{W}^{\mu} \widetilde{W}^{\nu}+\widetilde{W}^{\nu} \widetilde{W}^{\mu}\right] / 2$ and $\left[\widetilde{W}^{\mu} \widetilde{P}_{A}^{\nu}+\widetilde{W}^{\nu} \widetilde{P}_{A}^{\mu}\right](Q \cdot W) / 2$ with $\widetilde{W}:=W-\left(Q \cdot W / Q^{2}\right) Q$ were permissible. Furthermore, the structure functions which augment the basic tensors depend on the Lorentz scalars $Q^{2}, Q \cdot P_{A}$ and $(Q \cdot W)^{2}$, where the dependence on $(Q \cdot W)^{2}$ is at most linear and could therefore explicitly be split off. This strategy would result in a perfectly admissible and absolutely general parametrization of the current tensor. However, those additional terms, specific for a spin- 1 target, are not traceless and therefore would yield a nonstandard parametrization upon spin averaging. In contrast, the modified, though clumsier basic tensor forms in Eq. (B1):

$$
G^{\mu \nu}(W)=\left[g^{\mu}{ }_{\mu^{\prime}}-\frac{Q^{\mu} Q_{\mu^{\prime}}}{Q^{2}}\right] T^{\mu^{\prime} \nu^{\prime}}(W)\left[g_{\nu^{\prime}}{ }^{\nu}-\frac{Q_{\nu^{\prime}} Q^{\nu}}{Q^{2}}\right],
$$




$$
\begin{aligned}
H^{\mu \nu}(W)= & {\left[g^{\mu}{ }_{\mu^{\prime}}-\frac{Q^{\mu} Q_{\mu^{\prime}}}{Q^{2}}\right]\left[P_{A} Q_{\alpha} T^{\alpha \nu^{\prime}}(W)\right.} \\
& \left.+T^{\mu^{\prime} \alpha}(W) Q_{\alpha} P_{A}\right] \frac{1}{m_{A}^{2}}\left[g_{\nu^{\prime}}{ }^{\nu}-\frac{Q_{\nu^{\prime}} Q^{\nu}}{Q^{2}}\right]
\end{aligned}
$$

and the scalar $Q \cdot T(W) \cdot Q=Q_{\mu} T^{\mu \nu}(W) Q_{\nu}$ are traceless with respect to spin summation. Thus, the structure functions proportional to the Pauli-Lubanski vector $W$, i.e., $g_{i}^{A}$, and the structure functions proportional to the basis tensors $G^{\mu \nu}(W)$ and $H^{\mu \nu}(W)$ and to the scalar $Q \cdot T(W) \cdot Q$, i.e., $b_{i}^{A}$, cannot contribute in the spin-averaged case. When spin averaging, the current tensor (B1) reduces to the well-known form with the two structure functions $F_{1}^{A}$ and $F_{2}^{A}$.

The current tensor $\left\langle n_{A}\left|W_{A}^{\mu \nu}\left(Q, P_{A}\right)\right| n_{A}\right\rangle$ follows from the current tensor operator $W_{A}^{\mu \nu}\left(Q, P_{A}\right)$ of Eq. (B1) and from the density operator $\rho_{A}\left(n_{A} P_{A}\right)$ of Eq. (A2) according to Eq. (3.2). In fact, the current tensor $\left\langle n_{A}\left|W_{A}^{\mu \nu}\left(Q, P_{A}\right)\right| n_{A}\right\rangle$ is obtained from the operator (B1) by replacing the PauliLubanski vector $W$ and the tensor operator $T^{\mu \nu}(W)$ according to the relations

$$
\begin{gathered}
n_{A}^{\mu}=\left(1 / j m_{A}\right) \operatorname{Tr}\left[W^{\mu} \rho_{A}\left(n_{A}, P_{A}\right)\right], \\
t_{A}^{\mu \nu}=\left(1 / m_{A}^{2}\right) \operatorname{Tr}\left[T^{\mu \nu}(W) \rho_{A}\left(n_{A} P_{A}\right)\right] .
\end{gathered}
$$

We note that Eq. (B4a) differs from the corresponding Eq. (3.10) for a spin- $\frac{1}{2}$ target, since the quantum number $j$ is different; this difference is obvious, since polarization is defined with respect to the maximum angular momentum projection $j$; despite that difference the current tensors in Eqs. (3.8) and (B1) are defined such that their dependence on the polarization vector and on the spin-structure functions $g_{i}^{A}$ is the same for spin-1 and spin- $\frac{1}{2}$ targets.

\section{APPENDIX C: FRONT FORM MOMENTUM DENSITY OF THE DEUTERON AND SPIN DISTRIBUTION}

In this section the front form momentum density $\rho\left(\mathbf{p}_{N} t_{N}\right)$ of Eq. (5.8b) is calculated for the deuteron, i.e., for $A=2$. It is calculated as an operator in nucleonic and nuclear spin space. As in Eq. (6.2b) of Sec. VI it is related to the nuclear bound state $\left|\mathbf{P}_{A} \lambda_{A}\right\rangle$ with spin projection $\lambda_{A}$ by

$$
\begin{aligned}
\left\langle\lambda_{N} \lambda_{A}^{\prime}\left|\rho\left(\mathbf{p}_{N} t_{N}\right)\right| \lambda_{N}^{\prime} \lambda_{A}\right\rangle & =\frac{A}{P_{A}^{+}}\left\langle\mathbf{P}_{A} \lambda_{A}^{\prime}\right| \delta\left(\frac{p_{N}^{+}}{P_{A}^{+}}-\frac{p_{1}^{+}}{P_{A}^{+}}\right) \delta^{2}\left(\mathbf{p}_{N \perp}-\mathbf{p}_{1 \perp}\right) \\
& \times O_{1}\left(\lambda_{N}^{\prime} \lambda_{N}\right) \delta_{t_{N} t_{1}}\left|\mathbf{P}_{A} \lambda_{A}\right\rangle
\end{aligned}
$$

The nuclear c.m. system is assumed for the calculation. The internal part of the nuclear bound state $\left|\mathbf{P}_{A} \lambda_{A}\right\rangle$ is a mass eigenstate and it can therefore be identified with the nonrelativistic ground state $\left|\Psi_{B} \lambda_{A}\right\rangle$ of internal motion. It is calculated in terms of the three-dimensional relative momentum $\vec{p}$ and in terms of canonical spins, but has now to be transformed to the appropriate front form variables.

Internal single-nucleon front form momenta $\left(\mathbf{k}_{i \perp} \xi_{i}\right)$ are introduced as in Sec. VI and extended to three-dimensional form there; the momenta are constrained, $\left(\mathbf{k}_{1 \perp} \xi_{1}\right)$ are the independent ones. The relative momentum $\vec{p}$ is expressed by them, i.e.,

$$
\begin{gathered}
\vec{p}=\frac{1}{2}\left(\vec{k}_{1}-\vec{k}_{2}\right), \\
\vec{p}=\left[k_{1}^{1}, k_{1}^{2}, \frac{1}{2}\left(\xi_{1} M_{0}-\frac{m_{N}^{2}+\mathbf{k}_{1 \perp}^{2}}{\xi_{1} M_{0}}\right)\right],
\end{gathered}
$$

with the abbreviation

$$
M_{0}=\left[\frac{m_{N}^{2}+\mathbf{k}_{1 \perp}^{2}}{\xi_{1}\left(1-\xi_{1}\right)}\right]^{1 / 2}
$$

The two-nucleon bound state wave function takes the following form in that representation:

$$
\left\langle\mathbf{k}_{1 \perp} \xi_{1} \lambda_{1} s_{2} t_{1} t_{2} \mid \Psi_{B} \lambda_{A}\right\rangle=\sum_{s_{1}}\left\langle\lambda_{1}\left|R_{M}^{+}\left(\mathbf{k}_{1 \perp} \xi_{1}\right)\right| s_{1}\right\rangle \sqrt{\frac{\partial \vec{p}}{\partial\left(\mathbf{k}_{1 \perp} \xi_{1}\right)}}\left\langle\vec{p}\left(\mathbf{k}_{1 \perp} \xi_{1}\right) s_{1} s_{2} t_{1} t_{2} \mid \Psi_{B} \lambda_{A}\right\rangle .
$$

The transformation of momenta requires the Jacobi determinant

$$
\left|\frac{\partial \vec{p}}{\partial\left(\mathbf{k}_{1 \perp} \xi_{1}\right)}\right|=\left|\frac{\partial p^{3}}{\partial \xi_{1}}\left(\mathbf{k}_{1 \perp} \xi_{1}\right)\right|=\frac{M_{0}\left(\mathbf{k}_{1 \perp} \xi_{1}\right)}{4 \xi_{1}\left(1-\xi_{1}\right)},
$$

the transformation of spin the Melosh rotation

$$
R_{M}\left(\mathbf{k}_{1 \perp} \xi_{1}\right)=\frac{m_{N}+\xi_{1} M_{0}+i\left(\sigma_{N}^{1} k_{1}^{2}-\sigma_{N}^{2} k_{1}^{1}\right)}{\sqrt{\left(m_{N}+\xi_{1} M_{0}\right)^{2}+\mathbf{k}_{1 \perp}^{2}}}
$$


in Eq. $(\mathrm{C} 3 \mathrm{~b})$ the argument list of $M_{0}$ is made explicit. The front form momentum density samples the front form properties of a single nucleon. Thus, only the spin of nucleon 1 is Melosh rotated. In contrast, the spin of nucleon 2 remains in canonical representation, since the expectation value in Eq. (C1) sums over that spin dependence. The front form momentum density takes the form

$$
\begin{aligned}
\left\langle\lambda_{N} \lambda_{A}^{\prime}\left|\rho\left(\mathbf{p}_{N} t_{N}\right)\right| \lambda_{N}^{\prime} \lambda_{A}\right\rangle= & \frac{A}{P_{A}^{+}} \sum_{t_{2}} \sum_{s_{1}^{\prime} s_{1} s_{2}}\left|\frac{\partial p^{3}\left(\mathbf{p}_{N \perp} \xi\right)}{\partial \xi}\right|\left\langle\lambda_{N}\left|R_{M}^{+}\left(\mathbf{p}_{N \perp} \xi\right)\right| s_{1}\right\rangle\left\langle s_{1}^{\prime}\left|R_{M}\left(\mathbf{p}_{N \perp} \xi\right)\right| \lambda_{N}^{\prime}\right\rangle\left\langle\Psi_{B} \lambda_{A}^{\prime} \mid \vec{p}\left(\mathbf{p}_{N \perp} \xi\right) s_{1}^{\prime} s_{2} t_{1} t_{2}\right\rangle \\
& \times\left.\left\langle\vec{p}\left(\mathbf{p}_{N \perp} \xi\right) s_{1} s_{2} t_{1} t_{2} \mid \Psi_{B} \lambda_{A}\right\rangle\right|_{\xi=p_{N}^{+} / P_{A}^{+} .}
\end{aligned}
$$

At this stage we go back to the corresponding instant form momentum density $\rho\left(\vec{p}_{N} t_{N}\right)$ which reads in the nuclear c.m. system

$$
\begin{aligned}
\left\langle s_{N} s_{A}^{\prime}\left|\rho\left(\vec{p}_{N} t_{N}\right)\right| s_{N}^{\prime} s_{A}\right\rangle= & \left\langle\Psi_{B} s_{A}^{\prime}\left|\sum_{i} \delta\left(\vec{p}_{N}-\vec{k}_{i}\right) O_{i}\left(s_{N}^{\prime} s_{N}\right) \delta_{t_{N} t_{i}}\right| \Psi_{B} s_{A}\right\rangle=A \sum_{t_{2}} \sum_{s_{2}}\left\langle\Psi_{B} s_{A}^{\prime} \mid \vec{p}_{N} s_{N}^{\prime} s_{2} t_{N} t_{2}\right\rangle\left\langle\vec{p}_{N} s_{N} s_{2} t_{N} t_{2} \mid \Psi_{B} s_{A}\right\rangle \\
= & \frac{1}{2}\left\langle s_{N} s_{A}^{\prime}\right| \rho_{0}\left(p_{N} t_{N}\right)+\rho_{1}\left(p_{N} t_{N}\right) \vec{\sigma}_{N} \cdot \vec{S}_{A}+\rho_{2}\left(p_{N} t_{N}\right)\left(\frac{\vec{\sigma}_{N} \cdot \vec{p}_{N} \vec{S}_{A} \cdot \vec{p}_{N}}{\vec{p}_{N}{ }^{2}}-\frac{1}{3} \vec{\sigma}_{N} \cdot \vec{S}_{A}\right) \\
& +\rho_{3}\left(p_{N} t_{N}\right) \sum_{M} T_{A M}^{[2]}\left(S_{A}\right) Y_{2 M}^{*}\left(\hat{p}_{N}\right)\left|s_{N}^{\prime} s_{A}\right\rangle .
\end{aligned}
$$

The first step gives the definition. In the next two steps of Eq. (C5) the spin structure of the instant form momentum density $\rho\left(\vec{p}_{N} t_{N}\right)$ is recalled. The spin operator $T_{A}^{[2]}\left(S_{A}\right)$ was already introduced in Appendix A; it is a five-component tensor of rank 2 and is related to its Cartesian form (A7) in standard fashion. In addition to the three functions $\rho_{0}, \rho_{1}$, and $\rho_{2}$, which are already present in the description of the spin- $\frac{1}{2}$ target, a fourth function $\rho_{3}$ occurs, which is responsible for the tensor polarization. The functions $\rho_{0}, \rho_{1}, \rho_{2}$, and $\rho_{3}$ of Eq. (C5) are numerically available and are used for calculating the front form momentum distribution. However, neither $\rho_{0}$ nor $\rho_{3}$ will affect the spin-structure functions $g_{1}^{A}$ and $g_{2}^{A}$; both determine the front form momentum distribution $f_{N}\left(\xi t_{N}\right)$ of Eq. (5.14a), required for the calculation of the spin-independent deuteron structure function $F_{2}^{A}$. Since the calculation is carried out in the nuclear c.m. system, the canonical and front form spins are identical, thus, $\left|\Psi_{B} \lambda_{A}\right\rangle=\left|\Psi_{B} s_{A}\right\rangle$ and therefore

$$
\begin{aligned}
& \left\langle\lambda_{N} \lambda_{A}^{\prime}\left|\rho\left(\mathbf{p}_{N} t_{N}\right)\right| \lambda_{N}^{\prime} \lambda_{A}\right\rangle=\left|\frac{1}{2} \frac{1}{P_{A}^{+}}\right| \frac{\partial p^{3}\left(\mathbf{p}_{N \perp} \xi\right)}{\partial \xi} \mid\left\langle\lambda_{N} \lambda_{A}^{\prime}\right| R_{M}^{+}\left(\mathbf{p}_{N \perp} \xi\right)\left[\rho_{0}\left(p t_{N}\right)+\rho_{1}\left(p t_{N}\right) \vec{\sigma}_{N} \cdot \vec{S}_{A}+\rho_{2}\left(p t_{N}\right)\left(\frac{\vec{\sigma}_{N} \cdot \vec{p} \vec{S}_{A} \cdot \vec{p}}{\vec{p}^{2}}\right.\right. \\
& \left.\left.-\frac{1}{3} \vec{\sigma}_{N} \cdot \vec{S}_{A}\right)+\sum_{M} T_{A M}^{[2]}\left(S_{A}\right) Y_{2 M}^{*}(\hat{p}) \rho_{3}\left(p t_{N}\right)\right]\left.\left.\right|_{\vec{p}=\vec{p}\left(\mathbf{p}_{N \perp} \xi\right)} R_{M}\left(\mathbf{p}_{N \perp} \xi\right)\left|\lambda_{N}^{\prime} \lambda_{A}\right\rangle\right|_{\xi=p_{N}^{+} / P_{A}^{+}} .
\end{aligned}
$$

The result (C6) requires the replacement of the Jacobi momentum $\vec{p}$ as $\vec{p}\left(\mathbf{p}_{N \perp} \xi\right)$ by the front form momenta ( $\left.\mathbf{p}_{N \perp} \xi\right)$ according to the prescription of Eq. $(\mathrm{C} 2 \mathrm{~b})$, given there in terms of $\left(\mathbf{k}_{1 \perp} \xi_{1}\right)$.

The determination of the spin-structure functions $g_{1}^{A}$ and $g_{2}^{A}$ in deep-inelastic lepton scattering according to Eqs. (5.19) requires the full spin-dependent momentum density $\rho\left(\mathbf{p}_{N} t_{N}\right)$; however, the determination according to Eqs. (5.12) needs the front form spin distribution $\vec{s}_{N}\left(\xi t_{N} n_{A}\right)$ only, i.e.,

$$
\vec{s}_{N}\left(\xi t_{N} n_{A}\right)=\left.\frac{1}{2} \int d^{2} \mathbf{p}_{N \perp}\left|\frac{\partial p^{3}}{\partial \xi}\left(\mathbf{p}_{N \perp} \xi\right)\right| \sum_{i=1}^{3} \operatorname{Tr}\left[\vec{\sigma}_{N} R_{M}^{+}\left(\mathbf{p}_{N \perp} \xi\right) \sigma_{N}^{i} R_{M}\left(\mathbf{p}_{N \perp} \xi\right)\right]\left[\rho_{1}\left(p t_{N}\right) n_{A}^{i}+\rho_{2}\left(p t_{N}\right)\left(\frac{p^{i} \vec{n}_{A} \cdot \vec{p}}{\vec{p}^{2}}-\frac{1}{3} n_{A}^{i}\right)\right]\right|_{\vec{p}=\vec{p}\left(\mathbf{p}_{N \perp} \xi\right)}
$$

The front form spin distribution $\vec{s}_{N}\left(\xi t_{N} n_{A}\right)$ was already introduced in Eq. (5.13). The calculations of this paper are based on it. It satisfies the sum rule (5.15) relating it to the spin expectation value $\left\langle\sigma_{N\left(t_{N}\right)}\right\rangle$ in the polarized deuteron state $\left|\Psi_{B} n_{A}\right\rangle$, defined by Eq. (5.16a). The spin expectation value $\left\langle\sigma_{N\left(t_{N}\right)}\right\rangle$ is given by the $S$ - and $D$-state probabilities of the two-nucleon bound state wave function according to

$$
\left\langle\sigma_{N\left(t_{N}\right)}\right\rangle=\frac{1}{2}\left(P(S)-\frac{1}{2} P(D)\right)
$$


[1] J. Friar, B.F. Gibson, G.L. Payne, A.M. Bernstein, and T.E. Chupp, Phys. Rev. C 42, 2310 (1990).

[2] E.W. Otten, Prog. Part. Nucl. Phys. 24, 103 (1990).

[3] R.-W. Schulze and P.U. Sauer, Phys. Rev. C 48, 38 (1993).

[4] C.E. Woodward et al., Phys. Rev. Lett. 65, 698 (1990).

[5] C.E. Woodward et al., Phys. Rev. C 44, R571 (1991).

[6] A.K. Thompson et al., Phys. Rev. Lett. 68, 2901 (1992).

[7] C.E. Jones et al., Phys. Rev. C 47, 110 (1993).

[8] H. Gao et al., Phys. Rev. C 50, R546 (1994).

[9] J.-O. Hansen et al., Phys. Rev. Lett. 74, 654 (1995).

[10] P.L. Anthony et al., Phys. Rev. Lett. 71, 959 (1993).

[11] D.W. Kawall, Ph.D. thesis, Stanford University, 1995 (unpublished).

[12] B. Adeva et al., Phys. Lett. B 302, 533 (1993).

[13] K. Abe et al., Phys. Rev. Lett. 75, 25 (1995).

[14] K. Abe et al., Phys. Rev. Lett. 76, 587 (1996).

[15] E.L. Berger and F. Coester, in Quarks and Gluons in Particles and Nuclei, edited by S. Brodsky and E. Moniz (World Scientific, Singapore, 1986), p. 255.

[16] B. Keister and W. Polyzou, Adv. Nucl. Phys. 20, 225 (1991).

[17] F. Coester, Prog. Part. Nucl. Phys. 29, 1 (1992).

[18] U. Oelfke, P.U. Sauer, and F. Coester, Nucl. Phys. A518, 583 (1990).

[19] J. Carbonell and V.A. Karmanov, Nucl. Phys. A581, 625 (1995).

[20] M. Lacombe, B. Loiseau, S.M. Richard, R. Vinh Mau, J.
Côté, P. Pirès, and R. de Tourreil, Phys. Rev. C 21, 861 (1980).

[21] Ch. Hajduk and P.U. Sauer, Nucl. Phys. A369, 321 (1981).

[22] A. Stadler, P.U. Sauer, and W. Glöckle, Phys. Rev. C 44, 2319 (1991); A. Stadler (private communication).

[23] A. Schaefer, Phys. Lett. B 208, 175 (1988).

[24] J. Ashman et al., Phys. Lett. B 206, 364 (1988); Nucl. Phys. B328, 1 (1989).

[25] J.D. Bjorken, Phys. Rev. 148, 1467 (1966); Phys. Rev. D 1, 1376 (1970).

[26] S. Wandzura and F. Wilczek, Phys. Lett. 72B, 195 (1977).

[27] J. Kaur, Nucl. Phys. B128, 219 (1977).

[28] E.L. Berger and F. Coester, Annu. Rev. Nucl. Part. Sci. 37, 463 (1987)

[29] S.V. Akulinichev, S.A. Kulagin, and G.M. Vagradov, Phys. Rev. B 158, 485 (1985).

[30] R.M. Woloshyn, Nucl. Phys. A496, 749 (1989).

[31] C. Ciofi degli Atti, E. Pace, and G. Salmè, Phys. Rev. C 46, R1591 (1992).

[32] C. Ciofi degli Atti, S. Scopetta, E. Pace, and G. Salmè, Phys. Rev. C 48, R968 (1993).

[33] L.P. Kaptare, A.Yu. Umnikov, C. Ciofi degli Atti, S. Scopetta, and K.Yu. Kazakov, Phys. Rev. C 51, 52 (1995).

[34] S.A. Kulagin, W. Melnitchouk, G. Pillar, and W. Weise, Phys. Rev. C 52, 932 (1995). 